\title{
A new type of solutions for a singularly perturbed elliptic Neumann problem
}

\section{Gongbao Li, Shuangjie Peng and Shusen Yan}

\begin{abstract}
We prove the existence of positive solutions concentrating simultaneously on some higher dimensional manifolds near and on the boundary of the domain for a nonlinear singularly perturbed elliptic Neumann problem.
\end{abstract}

\section{Introduction}

The aim of this paper is to construct solutions concentrating on some higher dimensional manifolds for the following singularly perturbed elliptic problem:

$$
\begin{cases}-\varepsilon^{2} \Delta u+u=u^{p-1}, u>0, & \text { in } \Omega \\ \frac{\partial u}{\partial n}=0, & \text { on } \partial \Omega\end{cases}
$$

where $\varepsilon>0$ is a small number, $\Omega$ is an open domain and $n$ is the outward unit normal of $\partial \Omega$ at $y \in \partial \Omega$.

We assume that $\Omega$ is a domain in $\mathbb{R}^{N}$, whose boundary is Lipschitz continuous, and satisfies the following condition:

$\left(\Omega_{1}\right)$ : there is an integer $m, 1<m<N$, such that $y \in \Omega$, if and only if $\left(\left|y^{\prime}\right|, y^{\prime \prime}\right) \in D$, where $y=\left(y^{\prime}, y^{\prime \prime}\right), y^{\prime} \in \mathbb{R}^{m}, y^{\prime \prime} \in \mathbb{R}^{N-m}, D$ is a relatively open domain in $\mathbb{R}_{+}^{N-m+1}$, and

$$
\mathbb{R}_{+}^{N-m+1}=\left\{z=\left(z_{1}, \ldots, z_{N-m+1}\right): z_{1} \geq 0\right\} .
$$

2000 Mathematics Subject Classification: 35J60, 35J25.

Keywords: Singularly perturbed elliptic equation; variational method, concentrating solutions, higher dimensional manifolds. 
In this paper, we do not assume that $\Omega$ is bounded. The domain $\Omega$ can be a bounded domain, or an exterior domain in $\mathbb{R}^{N}$, or many other unbounded domains.

We assume that $p$ satisfies

$$
\begin{aligned}
& p \in(2,2(N-m+1) /(N-m-1)) \quad \text { if } m<N-1, \\
& p \in(0,+\infty) \quad \text { if } m \geq N-1 .
\end{aligned}
$$

In view of the assumption on $\Omega$, we will work on the following subspace of $H^{1}(\Omega)$ :

$$
H_{s}=\left\{u: u \in H^{1}(\Omega), u(y)=u\left(\left|y^{\prime}\right|, y^{\prime \prime}\right)\right\} .
$$

Let $U$ be the unique solution of the following problem:

$$
\begin{cases}-\Delta v+v=v^{p-1}, v>0, & \text { in } \mathbb{R}^{N-m+1}, \\ v(0)=\max _{z \in \mathbb{R}^{N-m+1}} v(z), & v \in H^{1}\left(\mathbb{R}^{N-m+1}\right) .\end{cases}
$$

Then $U(z)=U(|z|), U^{\prime}<0$,

$$
|z|^{(N-m) / 2} e^{|z|} U(|z|) \rightarrow c>0,|z| \rightarrow+\infty,
$$

and $U(z)$ also satisfies

$$
\begin{cases}-\Delta v+v=v^{p-1}, v>0, & \text { in } \mathbb{R}_{+}^{N-m+1}, \\ v(0)=\max _{z \in \mathbb{R}_{+}^{N-m+1}} v(z), & v \in H^{1}\left(\mathbb{R}_{+}^{N-m+1}\right), \\ \frac{\partial v}{\partial x_{N-m+1}}=0, & x \in \partial \mathbb{R}_{+}^{N-m+1} .\end{cases}
$$

Furthermore, $U(z)$ is nondegenerate. That is, the kernel of the operator $-\Delta w+w-(p-1) U^{p-2} w$ in $H^{1}\left(\mathbb{R}^{N-m+1}\right)$ is spanned by

$$
\left\{\frac{\partial U(z)}{\partial z_{i}}, i=1, \cdots, N-m+1\right\}
$$

and the kernel of the operator $-\Delta w+w-(p-1) U^{p-2} w$ in $H^{1}\left(\mathbb{R}_{+}^{N-m+1}\right)$ with Neumann boundary condition $\frac{\partial w}{\partial x_{N-m+1}}=0$ is spanned by

$$
\left\{\frac{\partial U(z)}{\partial x_{i}}, i=1, \cdots, N-m\right\} .
$$

See $[15,23]$.

For any $y=\left(y^{\prime}, y^{\prime \prime}\right) \in \mathbb{R}^{N}, y^{\prime} \in \mathbb{R}^{m}, y^{\prime \prime} \in \mathbb{R}^{N-m}$, we denote $\tilde{y}=$ $\left(\left|y^{\prime}\right|, y^{\prime \prime}\right) \in \mathbb{R}^{N-m+1}$. Let $\bar{W}(y)=U(\tilde{y})$. For any $\bar{x} \in D$, let $\bar{W}_{\varepsilon, \bar{x}}(y)=$ $U(|\tilde{y}-\bar{x}| / \varepsilon)$. Then, $\bar{W}_{\varepsilon, \bar{x}}$ satisfies

$$
-\varepsilon^{2} \Delta \bar{W}_{\varepsilon, \bar{x}}+\bar{W}_{\varepsilon, \bar{x}}=\bar{W}_{\varepsilon, \bar{x}}^{p-1}-\varepsilon \frac{m-1}{\left|y^{\prime}\right|} \frac{\left|y^{\prime}\right|-\bar{x}_{1}}{|\tilde{y}-\bar{x}|} U^{\prime}\left(\frac{|\tilde{y}-\bar{x}|}{\varepsilon}\right), \quad \text { in } \Omega .
$$


In this paper, we assume that $\Omega$ also satisfies the following condition: $\left(\Omega_{2}\right)$ : there exists $\bar{x}=\left(\bar{x}_{1}, \bar{x}^{\prime \prime}\right) \in \partial D$, such that

(i) there is a $C^{2}$ function $\psi\left(z^{\prime \prime}\right)$ in $\mathbb{R}^{N-m}$, such that

$$
D \cap B_{\delta}(\bar{x})=\left\{z=\left(z_{1}, z^{\prime \prime}\right): z_{1}<\psi\left(z^{\prime \prime}\right)\right\} \cap B_{\delta}(\bar{x}),
$$

and

$$
\partial D \cap B_{\delta}(\bar{x})=\left\{z=\left(z_{1}, z^{\prime \prime}\right): z_{1}=\psi\left(z^{\prime \prime}\right)\right\} \cap B_{\delta}(\bar{x}),
$$

where $\delta>0$ is a constant;

(ii) $\bar{x}_{1}=\psi\left(\bar{x}^{\prime \prime}\right)=\max _{z^{\prime \prime} \in B_{\delta}\left(\bar{x}^{\prime \prime}\right)} \psi\left(z^{\prime \prime}\right)>0$, and $\bar{x}_{1}>\max _{z^{\prime \prime} \in \partial B_{\delta}\left(\bar{x}^{\prime \prime}\right)} \psi\left(z^{\prime \prime}\right)$.

We will prove that for any positive integer pair $\left(k_{1}, k_{2}\right),(1.1)$ has a solution $u_{\varepsilon}$, which is close to $\sum_{j=1}^{k_{1}+k_{2}} \bar{W}_{\varepsilon, x_{j}}$ in a small neighbourhood of $\left|y^{\prime}\right|=\bar{x}_{1}$ with $x_{j} \in D$ for $j=1, \ldots, k_{1}$ and $x_{j} \in \partial D$ for $j=k_{1}, \ldots, k_{2}$, and is close to zero elsewhere. Since the right hand side of (1.2) has a singularity at $y^{\prime}=0$, we truncate $\bar{W}_{\varepsilon, \bar{x}}$ as follows.

Let $\xi \in C_{0}^{\infty}\left(\mathbb{R}^{N-m+1}\right)$ be a function such that $\xi=0$ if $z_{1} \leq \kappa, \xi=1$ if $z_{1} \geq 2 \kappa$, for some small $\kappa>0$. For any $x_{j} \in D$ with $x_{j, 1} \geq 6 \kappa$, define

$$
W_{\varepsilon, x_{j}}(y)=\xi\left(\left|y^{\prime}\right|, y^{\prime \prime}\right) \bar{W}_{\varepsilon, x_{j}}(y) \text {. }
$$

Then $W_{\varepsilon, x_{j}}$ satisfies

$$
-\varepsilon^{2} \Delta W_{\varepsilon, x_{j}}+W_{\varepsilon, x_{j}}=\xi \bar{W}_{\varepsilon, x_{j}}^{p-1}+\tilde{f}_{\varepsilon, x_{j}}(y) \text { in } \Omega,
$$

where

$$
\begin{aligned}
\tilde{f}_{\varepsilon, x_{j}}(y)=- & \xi \varepsilon \frac{m-1}{\left|y^{\prime}\right|} \frac{\left|y^{\prime}\right|-x_{j, 1}}{\left|\tilde{y}-x_{j}\right|} U^{\prime}\left(\frac{\left|\tilde{y}-x_{j}\right|}{\varepsilon}\right)-2 \varepsilon D \xi D U\left(\frac{\left|\tilde{y}-x_{j}\right|}{\varepsilon}\right) \\
& -\varepsilon^{2} U\left(\frac{\left|\tilde{y}-x_{j}\right|}{\varepsilon}\right) \Delta \xi
\end{aligned}
$$

Since

$$
\frac{z_{1}-x_{j, 1}}{\left|z-x_{j}\right|} U^{\prime}\left(\frac{\left|z-x_{j}\right|}{\varepsilon}\right)=\varepsilon \frac{\partial}{\partial z_{1}} U\left(\frac{\left|z-x_{j}\right|}{\varepsilon}\right),
$$

and $\xi=0$ in a neighbourhood of $\left|y^{\prime}\right|=0$, it is easy to see that $\tilde{f}_{\varepsilon, x_{j}}$ is a smooth function in both $y$ and $x_{j}$, and satisfies

$$
\left|\tilde{f}_{\varepsilon, x_{j}}\right| \leq C \varepsilon U\left(\frac{\left|\tilde{y}-x_{j}\right|}{\varepsilon}\right) .
$$


Let $P_{\varepsilon, \Omega} W_{\varepsilon, x_{j}}$ be the solution of

$$
\begin{cases}-\varepsilon^{2} \Delta v+v=\xi \bar{W}_{\varepsilon, x_{j}}^{p-1}+\tilde{f}_{\varepsilon, x_{j}}(y), & \text { in } \Omega \\ \frac{\partial v}{\partial n}=0, & \text { on } \partial \Omega .\end{cases}
$$

By the uniqueness, we know that $P_{\varepsilon, \Omega} W_{\varepsilon, x_{j}} \in H_{s}$.

Let

$$
\langle u, v\rangle_{\varepsilon}=\int_{\Omega}\left(\varepsilon^{2} D u D v+u v\right), \quad\|v\|_{\varepsilon}=\langle u, v\rangle_{\varepsilon}^{1 / 2} .
$$

The main result of this paper is the following.

Theorem 1.1. Assume that $1<m<N$. Suppose that $\Omega$ satisfies $\left(\Omega_{1}\right)$ and $\left(\Omega_{2}\right)$. Then, for any positive integers $k_{1}$ and $k_{2}$, there is an $\varepsilon_{0}>0$, such that for every $\varepsilon \in\left(0, \varepsilon_{0}\right]$, (1.1) has a solution of the form

$$
u_{\varepsilon}=\sum_{j=1}^{k_{1}+k_{2}} P_{\varepsilon, \Omega} W_{\varepsilon, x_{\varepsilon, j}}+\omega_{\varepsilon}
$$

with $\omega_{\varepsilon} \in H_{s}$, where for $j=1, \ldots, k_{1}, x_{\varepsilon, j}=\left(x_{\varepsilon, j, 1}, x_{\varepsilon, j}^{\prime \prime}\right) \in D$, and as $\varepsilon \rightarrow 0$,

$$
\frac{d\left(x_{\varepsilon, j}, \partial D\right)}{\varepsilon} \rightarrow+\infty
$$

For $j=k_{1}+1, \ldots, k_{1}+k_{2}, x_{\varepsilon, j}=\left(x_{\varepsilon, j, 1}, x_{\varepsilon, j}^{\prime \prime}\right) \in \partial D$.

Moreover, for $i, j=1, \ldots, k_{1}+k_{2}$,

$$
\begin{gathered}
\frac{\left|x_{\varepsilon, j}-x_{\varepsilon, i}\right|}{\varepsilon} \rightarrow+\infty, \forall j \neq i, \\
x_{\varepsilon, j} \rightarrow \hat{x}_{j}=\left(\hat{x}_{j, 1}, \hat{x}_{j}^{\prime \prime}\right) \in \partial D \cap B_{\delta}(\bar{x}), \quad \text { with } \hat{x}_{j, 1}=\psi\left(\hat{x}_{j}^{\prime \prime}\right)=\max _{z^{\prime \prime} \in B_{\delta}\left(\bar{x}^{\prime \prime}\right)} \psi\left(z^{\prime \prime}\right),
\end{gathered}
$$

and

$$
\left\|\omega_{\varepsilon}\right\|_{\varepsilon}^{2}=o\left(\varepsilon^{N-m+1}\right) .
$$

In $[18,19]$, Malchiodi and Montenegro obtained solutions concentrating on higher dimensional subsets of the boundary, which seems to be the first results concerning solutions concentrating on higher-dimensional sets.

For (1.1) with the Dirichlet boundary condition, the results in $[7,10]$ show that it has a solution concentrating on a manifold near $\left|y^{\prime}\right|=x_{1}$, where $x_{1}>0$ is a local minimum of the distance of $x$ to $\left\{z_{1}=0\right\}$ for $x \in D$.

For the Neumann problem, in [10], Dance and Yan constructed solutions concentrating on higher dimensional subsets inside the domain and on the boundary of the domain separately, and all the manifolds are close to $\left|y^{\prime}\right|=\hat{x}_{1}$, where $\hat{x}_{1}>0$ is a local maximum of the distance of $x$ to $\left\{z_{1}=0\right\}$ for $x \in D$. 
For problem (1.1) with potential functions $V(x)$ and $K(x)$ multiplying the linear term $u$ and nonlinear term $u^{p-1}$ respectively, Ambrosetti, Malchiodi and $\mathrm{Ni}[1,2]$ extended [18] to higher-dimensional spike-layers for problem (1.1) with $K(|x|) \equiv 1, \Omega=\mathbb{R}^{N}$ and $V(x)$ being radially symmetric.

Also, in [3, 4], Bartsch and Peng show that (1.1) has a solution concentrating on multi-dimensional subsets inside the domain, moreover, these results are true for both Dirichlet and Neumann boundary conditions.

For a survey of this kind of results we can also refer to [17].

Our result here shows that (1.1) has solutions concentrating simultaneously on several higher dimensional interior and boundary manifolds.

There are many works in the case $m=1$ since the pioneering works $[22,23]$. See for example $[5,8,9,11,12,13,14,16,24,25]$. To obtain the results mentioned above for the case $m=1$, no symmetry condition is imposed on the domain $\Omega$.

In the case $m>1$, we use the solution $U$ of a lower dimensional problem as an approximate solution for problem (1.1). So, there is no control in some directions for the corresponding linear operator

$$
L_{\varepsilon} v=:-\varepsilon^{2} \Delta v+v-(p-1) \bar{W}_{\varepsilon, \bar{x}}^{p-2} v \quad \text { in } \quad H^{1}(\Omega) .
$$

As a consequence, $L_{\varepsilon} v=\lambda v, v \in H^{1}(\Omega)$, will have many small eigenvalues. This is the main reason that Malchiodi and Montenegro [18] could only prove the existence of solutions concentrating on a whole connected component of $\partial \Omega$ for a sequence of $\varepsilon_{j} \rightarrow 0$. By imposing some partial symmetry conditions on $\Omega$, we can get rid of the small eigenvalues if we work on the subspace $H_{s}$.

The functional corresponding to (1.1) may not be well defined in $H_{s}$, because the exponent $p$ may be supercritical.

Our objective is to construct solutions concentrating near the $m-1$ dimensional manifolds $\left|y^{\prime}\right|=\bar{x}_{1}$. So we can modify the nonlinear term $u^{p-1}$ in such a way that corresponding to the modified problem, the functional is well defined in $H_{s}$, and the modified problem has a solution concentrating near $\left|y^{\prime}\right|=\bar{x}_{1}$, which is also a solution of the original problem. To this aim, we define

$$
f(y, t)=1_{B} t_{+}^{p-1}+\left(1-1_{B}\right) \bar{f}(t),
$$

where $B=\left\{y: y \in \Omega,\left(\left|y^{\prime}\right|, y^{\prime \prime}\right) \in D \cap B_{\delta}(\bar{x}) \subset\left\{\left(\left|y^{\prime}\right|, y^{\prime \prime}\right):\left|y^{\prime}\right| \geq \kappa\right\}\right\}, 1_{B}=1$ in $B$, and is zero otherwise, and

$$
\bar{f}(t)= \begin{cases}t_{+}^{p-1}, & t \leq 1 \\ 1+(p-1)(t-1), & t>1\end{cases}
$$


Now we consider the following problem:

$$
\begin{cases}-\varepsilon^{2} \Delta u+u=f(y, u), u>0, & \text { in } \Omega \\ u=0, & \text { on } \partial \Omega .\end{cases}
$$

The functional corresponding to (1.7) is

$$
I_{\varepsilon}(u)=\frac{1}{2} \int_{\Omega}\left(\varepsilon^{2}|D u|^{2}+u^{2}\right)-\int_{\Omega} F(y, u),
$$

where $F(y, t)=\int_{0}^{t} f(y, \tau) d \tau$. For any $y \in B$, we have $\left|y^{\prime}\right| \geq \kappa>0$. We see that $I_{\varepsilon}(u)$ is well defined in $H_{s}$ if $p \in(2,2(N-m+1) /(N-m-1))$.

Remark 1.1. After this work was completed, the paper [20] was published. In [20], $\Omega$ is supposed to be a unit ball $B_{1}(0)$ in $\mathbb{R}^{N}$ and a solution concentrating on several spheres $S_{r_{j, \varepsilon}}^{N-1}$ was constructed, where $S_{r_{j, \varepsilon}}^{N-1} \subset B_{1}(0)$ is an $(N-1)$-dimensional sphere with radius $r_{j, \varepsilon}$ and $r_{j, \varepsilon} \rightarrow 1$ as $\varepsilon \rightarrow 0$. We point out here that if $\Omega=B_{1}(0)$, our result shows that for any integer $1 \leq k<N-1$, there exists a solution concentrating on several $k$-dimensional spheres which can be simultaneously in $B_{1}(0)$ and on $\partial B_{1}(0)$, and all the radii of these spheres tent to 1 as $\varepsilon \rightarrow 0$. Hence our result gives a positive answer to the conjecture proposed by $\mathrm{Ni}$ in [21].

\section{Basic Estimates}

In this section, we give some basic estimates needed in the proof of the main result, under the assumption that $x_{j} \in D, x_{j, 1} \geq 6 \kappa, d\left(x_{j}, \partial D\right) / \varepsilon$ is large and $d\left(x_{j}, \partial D\right)$ is small. We assume that $d\left(x_{j}, \partial D\right)$ is small enough such that for $x_{j} \in D$ with $x_{j, 1} \geq 6 \kappa$,

$$
d\left(x_{j}, \partial B\right)=d\left(x_{j}, \partial D\right) .
$$

Let $\varphi_{\varepsilon, x_{j}}=W_{\varepsilon, x_{j}}-P_{\varepsilon, \Omega} U_{\varepsilon, x_{j}}$. Then $\varphi_{\varepsilon, x_{j}}$ satisfies

$$
\begin{cases}-\varepsilon^{2} \Delta \varphi_{\varepsilon, x_{j}}+\varphi_{\varepsilon, x_{j}}=0, & \text { in } \Omega \\ \frac{\partial \varphi_{\varepsilon, x_{j}}}{\partial n}=\frac{\partial W_{\varepsilon, x_{j}}}{\partial n}, & \text { on } \partial \Omega .\end{cases}
$$

Lemma 2.1. For any small $\theta>0$, there is a constant $C>0$, such that

$$
\left|\varphi_{\varepsilon, x}(y)\right| \leq \begin{cases}C \min \left\{e^{-d(x, \partial D) / \varepsilon} e^{-(1-\theta) d(\tilde{y}, \partial D) / \varepsilon}, e^{-(1-\theta)|\tilde{y}-x| / \varepsilon}\right\}, & x \in D, \\ C \varepsilon e^{-(1-\theta)|\tilde{y}-x| / \varepsilon}, & x \in \partial D .\end{cases}
$$


Proof. Let $G_{\varepsilon}(z, y)$ and $G(z, y)$ be the corresponding Green's functions of $-\varepsilon \Delta+I$ in $\Omega$ and $-\Delta+I$ in $\Omega_{\varepsilon, y}=\left\{z \in \mathbb{R}^{N}: \varepsilon z+y \in \Omega\right\}$ subject to the Neumann boundary condition respectively. Then

$$
G_{\varepsilon}(z, y)=\frac{1}{\varepsilon^{N}} G\left(\frac{z-y}{\varepsilon}, 0\right) .
$$

Suppose $x \in D$, then we have

$$
\begin{aligned}
\left|\varphi_{\varepsilon, x}(y)\right| & =\varepsilon^{2}\left|\int_{\partial \Omega} G_{\varepsilon}(z, y) \frac{\partial W_{\varepsilon, x}}{\partial n} d z\right| \leq C \varepsilon e^{-d(x, \partial D) / \varepsilon} \int_{\partial \Omega}\left|G_{\varepsilon}(z, y)\right| d y \\
& =C e^{-d(x, \partial D) / \varepsilon} \int_{\partial \Omega_{\varepsilon, y}}|G(z, 0)| d z \leq C e^{-d(x, \partial D) / \varepsilon} e^{-(1-\theta) d(\tilde{y}, \partial D) / \varepsilon},
\end{aligned}
$$

since $G(z, 0) \sim 1 /|z|^{N-2}$ as $|z| \rightarrow 0$ and $|G(z, 0)| \leq C e^{-|z|}$ as $|z| \rightarrow \infty$.

Since the solution of (2.1) is unique, we know $\varphi_{\varepsilon, x} \in H_{s}$. For any $y \in \Omega$, let $y^{*}=\left(\left|y^{\prime}\right|, 0, \ldots, 0, y^{\prime \prime}\right)$. Then

$$
\begin{aligned}
\left|z-y^{*}\right| & =\left(\left(z_{1}-\left|y^{\prime}\right|\right)^{2}+\sum_{l=2}^{m} z_{l}^{2}+\left|z^{\prime \prime}-y^{\prime \prime}\right|^{2}\right)^{1 / 2} \\
& \geq\left(\left(\left|z^{\prime}\right|-\left|y^{\prime}\right|\right)^{2}+\left|z^{\prime \prime}-y^{\prime \prime}\right|^{2}\right)^{1 / 2}=|\tilde{z}-\tilde{y}| .
\end{aligned}
$$

As a consequence,

$$
\begin{aligned}
\left|\varphi_{\varepsilon, x}(y)\right| & =\left|\varphi_{\varepsilon, x}\left(y^{*}\right)\right| \leq C \varepsilon \int_{\partial \Omega}\left|G_{\varepsilon}(z, y)\right| e^{-|\tilde{z}-x| / \varepsilon} d z \\
& =C \varepsilon^{1-N} \int_{\partial \Omega}\left|G\left(\frac{z-y^{*}}{\varepsilon}, 0\right)\right| e^{-|\tilde{z}-x| / \varepsilon} d z \\
& \leq C \varepsilon^{1-N} \int_{\partial \Omega} \frac{1}{\left(\frac{\left|z-y^{*}\right|}{\varepsilon}\right)^{N-2}} e^{-(1-\theta)\left|z-y^{*}\right| / \varepsilon} e^{-|\tilde{z}-x| / \varepsilon} d z \\
& \leq C \varepsilon^{1-N} e^{-(1-2 \theta)|\tilde{y}-x| / \varepsilon} \int_{\partial \Omega} \frac{1}{\left(\frac{\left|z-y^{*}\right|}{\varepsilon}\right)^{N-2}} e^{-2 \theta\left|z-y^{*}\right| / \varepsilon} d z \\
& \leq C e^{-(1-2 \theta)|\tilde{y}-x| / \varepsilon} .
\end{aligned}
$$

Suppose $x \in \partial D$, then

$$
\left|\frac{\partial W_{\varepsilon, x}}{\partial n}\right| \leq C \varepsilon e^{-(1-\theta)|\tilde{y}-x| / \varepsilon} .
$$

Using the same arguments as the case $x \in D$, we deduce

$$
\left|\varphi_{\varepsilon, x}(y)\right| \leq C \varepsilon e^{-(1-\theta)|\tilde{y}-x| / \varepsilon} .
$$

Hence we complete the proof. 
Lemma 2.2. Let $q \in[1, p-1)$ be a constant and suppose $x_{j} \in D, x_{i} \in \partial D$. Then there is a constant $\sigma>0$, such that

$$
\int_{\Omega} W_{\varepsilon, x_{j}}^{p-1-q}\left|\varphi_{\varepsilon, x_{j}}\right|^{q} W_{\varepsilon, x_{i}}=\varepsilon^{N-m+1} O\left(e^{-(2+\sigma) d_{j} / \varepsilon}+e^{-(1+\sigma)\left|x_{i}-x_{j}\right| / \varepsilon}\right) .
$$

Proof. We have

$$
\int_{\Omega} W_{\varepsilon, x_{j}}^{p-1-q}\left|\varphi_{\varepsilon, x_{j}}\right|^{q} W_{\varepsilon, x_{i}}=c_{m-1} \int_{D}\left|z_{1}\right|^{m-1} W_{\varepsilon, x_{j}}^{p-1-q}\left|\varphi_{\varepsilon, x_{j}}\right|^{q} W_{\varepsilon, x_{i}}
$$

Let $d_{i j}=\left|x_{i}-x_{j}\right|$. Let $\sigma^{\prime}>0$ be a fixed small constant. Then using Lemma 2.1, we obtain

$$
\begin{aligned}
& \int_{D \backslash B_{\sigma^{\prime} d_{i j}}\left(x_{j}\right)}\left|z_{1}\right|^{m-1} W_{\varepsilon, x_{j}}^{p-1-q}\left|\varphi_{\varepsilon, x_{j}}\right|^{q} W_{\varepsilon, x_{i}} \\
& \leq C \int_{D \backslash B_{\sigma^{\prime} d_{i j}}\left(x_{j}\right)} e^{-(p-1-\theta)\left|\tilde{y}-x_{j}\right| / \varepsilon} e^{-\left|\tilde{y}-x_{i}\right| / \varepsilon} \\
& \leq C e^{-d_{i j} / \varepsilon} \int_{D \backslash B_{\sigma^{\prime} d_{i j}}\left(x_{j}\right)} e^{-(p-2-\theta)\left|\tilde{y}-x_{j}\right| / \varepsilon} \\
& \leq C \varepsilon^{N-m+1} e^{-d_{i j} / \varepsilon-(p-2-\theta) d_{i j} / \varepsilon} \\
&=C \varepsilon^{N-m+1} e^{-(1+\sigma) d_{i j} / \varepsilon}
\end{aligned}
$$

Using Lemma 2.1 again, we obtain

$$
\begin{aligned}
\int_{B_{\sigma^{\prime} d_{i j}}\left(x_{j}\right)}\left|z_{1}\right|^{m-1} W_{\varepsilon, x_{j}}^{p-1-q}\left|\varphi_{\varepsilon, x_{j}}\right|^{q} W_{\varepsilon, x_{i}} \\
\leq C e^{-q d_{j} / \varepsilon} e^{-\left(1-\sigma^{\prime}\right) d_{i j} / \varepsilon} \int_{B_{\sigma^{\prime} d_{i j}}\left(x_{j}\right)} W_{\varepsilon, x_{j}}^{p-1-q} \\
\leq C \varepsilon^{N-m+1} e^{-q d_{j} / \varepsilon} e^{-\left(1-\sigma^{\prime}\right) d_{i j} / \varepsilon} \\
\leq C \varepsilon^{N-m+1}\left(e^{-(2+\sigma) d_{j} / \varepsilon}+e^{-(1+\sigma)\left|x_{i}-x_{j}\right| / \varepsilon}\right)
\end{aligned}
$$

Combining (2.2) and (2.3), we prove this lemma.

Lemma 2.3. For $x_{j} \in \partial D$ and $x_{i} \in D$, there is a constant $c_{0}>0$, such that

$$
\left|\int_{\Omega} W_{\varepsilon, x_{j}}^{p-1} P_{\varepsilon, \Omega} W_{\varepsilon, x_{i}}\right|=c_{0} U\left(\frac{\left|x_{i}-x_{j}\right|}{\varepsilon}\right) \varepsilon^{N-m+1}+O\left(\varepsilon^{N-m+2}\right) .
$$


Proof. By (1.3), (1.4) and Lemma 2.1, we have

$$
\begin{aligned}
& \int_{\Omega} W_{\varepsilon, x_{j}}^{p-1} P_{\varepsilon, \Omega} W_{\varepsilon, x_{i}} \\
& =\int_{\Omega} \xi \bar{W}_{\varepsilon, x_{j}}^{p-1} P_{\varepsilon, \Omega} W_{\varepsilon, x_{i}}+O\left(e^{-\sigma / \varepsilon}\right) \varepsilon^{N-m+1} \\
& =\int_{\Omega}\left(-\varepsilon^{2} \Delta W_{\varepsilon, x_{j}}+W_{\varepsilon, x_{j}}-\tilde{f}_{\varepsilon, x_{j}}(y)\right) P_{\varepsilon, \Omega} W_{\varepsilon, x_{i}}+O\left(e^{-\sigma / \varepsilon}\right) \varepsilon^{N-m+1} \\
& =\int_{\partial \Omega}\left(-\varepsilon^{2} \frac{\partial W_{\varepsilon, x_{j}}}{\partial n} P_{\varepsilon, \Omega} W_{\varepsilon, x_{i}}+\varepsilon^{2} \frac{\partial P_{\varepsilon, \Omega} W_{\varepsilon, x_{i}}}{\partial n} W_{\varepsilon, x_{j}}\right) \\
& \quad+O\left(e^{-d_{j} / \varepsilon} \varepsilon^{N-m+2}+\varepsilon^{N-m+2}\right)+\int_{\Omega}\left(-\varepsilon^{2} \Delta P_{\varepsilon, \Omega} W_{\varepsilon, x_{i}}+P_{\varepsilon, \Omega} W_{\varepsilon, x_{i}}\right) W_{\varepsilon, x_{j}} \\
& =\int_{\partial \Omega}-\varepsilon^{2} \frac{\partial W_{\varepsilon, x_{j}}}{\partial n} P_{\varepsilon, \Omega} W_{\varepsilon, x_{i}}+\int_{\Omega} \xi \bar{W}_{\varepsilon, x_{i}}^{p-1} W_{\varepsilon, x_{j}}+O\left(e^{-d_{j} / \varepsilon} \varepsilon^{N-m+2}+\varepsilon^{N-m+2}\right) \\
& =\int_{\partial \Omega}-\varepsilon^{2} \frac{\partial W_{\varepsilon, x_{j}}}{\partial n} P_{\varepsilon, \Omega} W_{\varepsilon, x_{i}}+\int_{\Omega} W_{\varepsilon, x_{i}}^{p-1} W_{\varepsilon, x_{j}}+O\left(e^{-d_{j} / \varepsilon} \varepsilon^{N-m+2}+\varepsilon^{N-m+2}\right) .
\end{aligned}
$$

Since $x_{j} \in \partial \Omega$, we have

$$
\left|\frac{\partial W_{\varepsilon, x_{j}}}{\partial n}\right| \leq C \varepsilon e^{-(1-\theta)\left|\tilde{y}-x_{j}\right| / \varepsilon}
$$

As a result, from Lemma 2.1, we conclude

$$
\left|\int_{\Omega} W_{\varepsilon, x_{j}}^{p-1} P_{\varepsilon, \Omega} W_{\varepsilon, x_{i}}\right|=c_{0} U\left(\frac{\left|x_{i}-x_{j}\right|}{\varepsilon}\right) \varepsilon^{N-m+1}+O\left(\varepsilon^{N-m+2}\right) .
$$

Lemma 2.4. Let $q \in[1, p-1)$ be a constant and suppose that $x_{j} \in \bar{D}$, $x_{h} \in \bar{D}$ and $x_{i} \in \bar{D}$ are different mutually. Then there is a constant $\sigma>0$, such that

$$
\begin{aligned}
& \left|\int_{\Omega} P_{\varepsilon, \Omega} W_{\varepsilon, x_{j}}^{p-1-q} P_{\varepsilon, \Omega} W_{\varepsilon, x_{h}}^{q} P_{\varepsilon, \Omega} W_{\varepsilon, x_{i}}\right| \\
& \quad \leq C \varepsilon^{N-m+1}\left(e^{-(1+\sigma)\left|x_{i}-x_{j}\right| / \varepsilon}+e^{-(1+\sigma)\left|x_{i}-x_{h}\right| / \varepsilon}+e^{-(1+\sigma)\left|x_{j}-x_{h}\right| / \varepsilon}\right) .
\end{aligned}
$$

Proof. We only prove the case $x_{j} \in \partial D, x_{h} \in \partial D$ and $x_{h} \in D$, the remaining cases are similar.

Without loss of generality, suppose that

$$
d_{i h}:=\left|x_{h}-x_{i}\right| \leq \min \left\{\left|x_{i}-x_{j}\right|,\left|x_{h}-x_{j}\right|\right\} .
$$


Let $\sigma^{\prime}$ be a small constant. By Lemma 2.1, we deduce

$$
\begin{aligned}
& \left|\int_{\Omega} P_{\varepsilon, \Omega} W_{\varepsilon, x_{j}}^{p-1-q} P_{\varepsilon, \Omega} W_{\varepsilon, x_{h}}^{q} P_{\varepsilon, \Omega} W_{\varepsilon, x_{i}}\right| \leq C \int_{D} W_{\varepsilon, x_{j}}^{p-1-q} W_{\varepsilon, x_{h}}^{q} e^{-(1-\theta)\left|\tilde{y}-x_{i}\right| / \varepsilon} \\
& =\int_{D \cap B_{\sigma^{\prime} d_{i h}\left(x_{j}\right)}} W_{\varepsilon, x_{j}}^{p-1-q} W_{\varepsilon, x_{h}}^{q} e^{-(1-\theta)\left|\tilde{y}-x_{i}\right| / \varepsilon} \\
& \quad+C \int_{D \backslash B_{\sigma^{\prime} d_{i h}\left(x_{j}\right)}} W_{\varepsilon, x_{j}}^{p-1-q} W_{\varepsilon, x_{h}}^{q} e^{-(1-\theta)\left|\tilde{y}-x_{i}\right| / \varepsilon} \\
& \quad \leq C e^{-\left(1-\sigma^{\prime}\right)\left|x_{h}\right| / \varepsilon} e^{-(1-\theta)\left|x_{i}-x_{j}\right| / \varepsilon} \int_{D} W_{\varepsilon, x_{j}}^{p-1-q} \\
& \quad+C e^{-(1-\theta)\left|x_{h}-x_{i}\right|} e^{-\sigma d_{i h} / \varepsilon} \int_{D} W_{\varepsilon, x_{j}}^{p-1-q-\theta^{\prime}} \\
& \leq C \varepsilon^{N-m+1}\left(e^{-(1+\sigma)\left|x_{i}-x_{j}\right| / \varepsilon}+e^{-(1+\sigma)\left|x_{i}-x_{h}\right| / \varepsilon}+e^{-(1+\sigma)\left|x_{j}-x_{h}\right| / \varepsilon}\right) .
\end{aligned}
$$

Proposition 2.1. Suppose that $x_{j} \in D \cap B_{\delta}(\bar{x})$ for $j=1, \cdots, k_{1}$ and $x_{j} \in \partial D \cap B_{\delta}(\bar{x})$ for $j=k_{1}+1, \ldots, k_{1}+k_{2}$. Then

$$
\begin{aligned}
I_{\varepsilon}\left(\sum_{j=1}^{k_{1}+k_{2}} P_{\varepsilon, \Omega} W_{\varepsilon, x_{j}}\right)= & A \varepsilon^{N-m+1} \sum_{j=1}^{k_{1}} x_{j, 1}^{m-1}+\frac{1}{2} A \varepsilon^{N-m+1} \sum_{j=k_{1}+1}^{k_{1}+k_{2}} x_{j, 1}^{m-1} \\
& +\frac{1}{2} \sum_{j=1}^{k_{1}} \tau_{\varepsilon, x_{j}}-c_{0} \varepsilon^{N-m+1} \sum_{1 \leq i<j \leq k_{1}+k_{2}} U\left(\frac{\left|x_{i}-x_{j}\right|}{\varepsilon}\right) \\
& +\varepsilon^{N-m+1} O\left(\sum_{j=1}^{k_{1}} e^{-(2+\sigma) d_{j} / \varepsilon}+\sum_{j \neq i} e^{-(1+\sigma)\left|x_{i}-x_{j}\right| / \varepsilon}+\varepsilon\right),
\end{aligned}
$$

where $c_{0}>0$ is a constant, $\sigma>0$ is a small constant,

$$
A=\left(\frac{1}{2}-\frac{1}{p}\right) c_{m-1} \int_{\mathbb{R}^{N-m+1}} U^{p}
$$

$\left(c_{m-1}\right.$ is the area of the unit sphere in $\left.\mathbb{R}^{m}\right)$, and for $j=1, \ldots, k_{1}$,

$$
\tau_{\varepsilon, x_{j}}=\int_{\Omega} \xi \bar{W}_{\varepsilon, x_{j}}^{p-1} \varphi_{\varepsilon, x_{j}}
$$

satisfying

$$
\begin{aligned}
C_{1} \varepsilon^{N-m+1} & e^{-(2+\theta) d_{j} / \varepsilon}+\varepsilon^{N-m+1} O\left(\varepsilon e^{-d_{j} / \varepsilon}\right) \\
& \leq-\tau_{\varepsilon, x_{j}} \leq C_{2} \varepsilon^{N-m+1} e^{-(2-\theta) d_{j} / \varepsilon}+\varepsilon^{N-m+1} O\left(\varepsilon e^{-d_{j} / \varepsilon}\right)
\end{aligned}
$$


Proof.

$$
\begin{aligned}
I_{\varepsilon} & \left(\sum_{j=1}^{k_{1}+k_{2}} P_{\varepsilon, \Omega} W_{\varepsilon, x_{j}}\right)=I_{\varepsilon}\left(\sum_{j=1}^{k_{1}} P_{\varepsilon, \Omega} W_{\varepsilon, x_{j}}\right)+I_{\varepsilon}\left(\sum_{j=k_{1}+1}^{k_{1}+k_{2}} P_{\varepsilon, \Omega} W_{\varepsilon, x_{j}}\right) \\
& +\sum_{i=1}^{k_{1}} \sum_{j=k_{1}+1}^{k_{1}+k_{2}}\left\langle P_{\varepsilon, \Omega} W_{\varepsilon, x_{i}}, P_{\varepsilon, \Omega} W_{\varepsilon, x_{j}}\right\rangle_{\varepsilon} \\
(2.5) \quad & -\frac{1}{p} \int_{\Omega}\left(\left(\sum_{j=1}^{k_{1}+k_{2}} P_{\varepsilon, \Omega} W_{\varepsilon, x_{j}}\right)_{+}^{p}-\left(\sum_{j=1}^{k_{1}} P_{\varepsilon, \Omega} W_{\varepsilon, x_{j}}\right)_{+}^{p}-\left(\sum_{j=k_{1}+1}^{k_{1}+k_{2}} P_{\varepsilon, \Omega} W_{\varepsilon, x_{j}}\right)_{+}\right) .
\end{aligned}
$$

Using the inequality

$$
(a+b)_{+}^{p}-a_{+}^{p}-b_{+}^{p}-p a_{+}^{p-1} b-p b_{+}^{p-1} a= \begin{cases}O\left(|a|^{p / 2} \mid b^{p / 2}\right), & 2<p \leq 4, \\ O\left(|a|^{p-2} b^{2}+|b|^{p-2} a^{2}\right), & p>4,\end{cases}
$$

we obtain

$$
\begin{aligned}
\frac{1}{p} \int_{\Omega}\left(\left(\sum_{j=1}^{k_{1}+k_{2}} P_{\varepsilon, \Omega} W_{\varepsilon, x_{j}}\right)_{+}^{p}-\left(\sum_{j=1}^{k_{1}} P_{\varepsilon, \Omega} W_{\varepsilon, x_{j}}\right)_{+}^{p}-\left(\sum_{j=k_{1}+1}^{k_{1}+k_{2}} P_{\varepsilon, \Omega} W_{\varepsilon, x_{j}}\right)_{+}\right) \\
=\int_{\Omega}\left(\sum_{i=1}^{k_{1}} P_{\varepsilon, \Omega} W_{\varepsilon, x_{i}}\right)_{+}^{p-1}\left(\sum_{j=k_{1}+1}^{k_{1}+k_{2}} P_{\varepsilon, \Omega} W_{\varepsilon, x_{j}}\right) \\
+\int_{\Omega}\left(\sum_{i=1}^{k_{1}} P_{\varepsilon, \Omega} W_{\varepsilon, x_{i}}\right)_{+}\left(\sum_{j=k_{1}+1}^{k_{1}+k_{2}} P_{\varepsilon, \Omega} W_{\varepsilon, x_{j}}\right)_{+}^{p-1} \\
+O\left(\int_{\Omega}\left(\sum_{i=1}^{k_{1}}\left|P_{\varepsilon, \Omega} W_{\varepsilon, x_{i}}\right|\right)^{1+\sigma}\left(\sum_{j=k_{1}+1}^{k_{1}+k_{2}}\left|P_{\varepsilon, \Omega} W_{\varepsilon, x_{i}}\right|\right)^{1+\sigma}\right),
\end{aligned}
$$

where $\sigma>0$ is a constant.

Using Lemma 2.1, Lemma 2.2 and Lemma 2.4, we see that,

$$
\begin{aligned}
& \mid \sum_{i=1}^{k_{1}} \sum_{j=k_{1}+1}^{k_{1}+k_{2}}\left\langle P_{\varepsilon, \Omega} W_{\varepsilon, x_{i}}, P_{\varepsilon, \Omega} W_{\varepsilon, x_{j}}\right\rangle_{\varepsilon} \\
& \quad-\int_{\Omega}\left(\sum_{i=1}^{k_{1}} P_{\varepsilon, \Omega} W_{\varepsilon, x_{i}}\right)_{+}^{p-1}\left(\sum_{j=k_{1}+1}^{k_{1}+k_{2}} P_{\varepsilon, \Omega} W_{\varepsilon, x_{j}}\right) \mid \\
& =\sum_{i=1}^{k_{1}} \sum_{j=k_{1}+1}^{k_{1}+k_{2}}\left\{\int_{\Omega}\left|\xi \bar{W}_{\varepsilon, x_{i}}^{p-1}+\tilde{f}_{\varepsilon, x_{i}}-\left(P_{\varepsilon, \Omega} W_{\varepsilon, x_{i}}\right)_{+}^{p-1}\right|\left|P_{\varepsilon, \Omega} W_{\varepsilon, x_{j}}\right|\right. \\
& \left.\quad+O\left(\varepsilon^{N-m+1} e^{-(1+\sigma)\left|x_{i}-x_{j}\right| / \varepsilon}\right)\right\}
\end{aligned}
$$




$$
\begin{aligned}
& \leq C \sum_{i=1}^{k_{1}} \sum_{j=k_{1}+1}^{k_{1}+k_{2}}\left\{\int_{\Omega} W_{\varepsilon, x_{i}}^{p-1-q}\left|\varphi_{\varepsilon, x_{i}}\right|{ }^{q} W_{\varepsilon, x_{j}}+O\left(\varepsilon^{N-m+1} e^{-(1+\sigma)\left|x_{i}-x_{j}\right| / \varepsilon}\right)\right\} \\
& \quad+O\left(\varepsilon^{N-m+2}\right) \\
& =\varepsilon^{N-m+1} O\left(\sum_{i=1}^{k_{1}} e^{-(2+\sigma) d_{i} / \varepsilon}+\sum_{i=1}^{k_{1}} \sum_{j=k_{1}+1}^{k_{1}+k_{2}} e^{-(1+\sigma)\left|x_{i}-x_{j}\right| / \varepsilon}+\varepsilon\right) .
\end{aligned}
$$

Using Lemma 2.1,

$$
\begin{gathered}
O\left(\int_{\Omega}\left(\sum_{i=1}^{k_{1}}\left|P_{\varepsilon, \Omega} W_{\varepsilon, x_{i}}\right|\right)^{1+\sigma}\left(\sum_{j=k_{1}+1}^{k_{1}+k_{2}}\left|P_{\varepsilon, \Omega} W_{\varepsilon, x_{j}}\right|\right)^{1+\sigma}\right) \\
\leq C \sum_{i=1}^{k_{1}} \sum_{j=k_{1}+1}^{k_{1}+k_{2}} \int_{\Omega}\left(W_{\varepsilon, x_{i}}^{1+\sigma} W_{\varepsilon, x_{j}}^{1+\sigma}+\left|\varphi_{\varepsilon, x_{i}}\right|^{1+\sigma} W_{\varepsilon, x_{j}}^{1+\sigma}+W_{\varepsilon, x_{i}}^{1+\sigma}\left|\varphi_{\varepsilon, x_{j}}\right|^{1+\sigma}\right. \\
\left.+\left|\varphi_{\varepsilon, x_{i}}\right|^{1+\sigma}\left|\varphi_{\varepsilon, x_{j}}\right|^{1+\sigma}\right) \\
=\varepsilon^{N-m+1} O\left(\sum_{i=1}^{k_{1}} \sum_{j=k_{1}+1}^{k_{1}+k_{2}} e^{-(1+\sigma)\left|x_{i}-x_{j}\right| / \varepsilon}\right) .
\end{gathered}
$$

From Lemma 2.1, Lemma 2.3 and Lemma 2.4, we see that

$$
\begin{aligned}
& \text { (2.9) } \int_{\Omega}\left(\sum_{i=1}^{k_{1}} P_{\varepsilon, \Omega} W_{\varepsilon, x_{i}}\right)_{+}\left(\sum_{j=k_{1}+1}^{k_{1}+k_{2}} P_{\varepsilon, \Omega} W_{\varepsilon, x_{j}}\right)_{+}^{p-1} \\
& =\sum_{i=1}^{k_{1}} \sum_{j=k_{1}+1}^{k_{1}+k_{2}}\left(P_{\varepsilon, \Omega} W_{\varepsilon, x_{i}}\right)_{+} W_{\varepsilon, x_{j}}^{p-1}+\varepsilon^{N-m+1} O\left(\sum_{i=1}^{k_{1}} \sum_{j=k_{1}+1}^{k_{1}+k_{2}} e^{-(1+\sigma)\left|x_{i}-x_{j}\right| / \varepsilon}+\varepsilon\right) \\
& =c_{0} \sum_{i=1}^{k_{1}} \sum_{j=k_{1}+1}^{k_{1}+k_{2}} U\left(\frac{\left|x_{i}-x_{j}\right|}{\varepsilon}\right)+\varepsilon^{N-m+1} O\left(\sum_{i=1}^{k_{1}} \sum_{j=k_{1}+1}^{k_{1}+k_{2}} e^{-(1+\sigma)\left|x_{i}-x_{j}\right| / \varepsilon}+\varepsilon\right) .
\end{aligned}
$$

Now combining (2.5)-(2.9), we obtain that

$$
\begin{aligned}
& I_{\varepsilon}\left(\sum_{j=1}^{k_{1}+k_{2}} P_{\varepsilon, \Omega} W_{\varepsilon, x_{j}}\right) \\
& =I_{\varepsilon}\left(\sum_{j=1}^{k_{1}} P_{\varepsilon, \Omega} W_{\varepsilon, x_{j}}\right)+I_{\varepsilon}\left(\sum_{j=k_{1}+1}^{k_{1}+k_{2}} P_{\varepsilon, \Omega} W_{\varepsilon, x_{j}}\right)-c_{0} \sum_{i=1}^{k_{1}} \sum_{j=k_{1}+1}^{k_{1}+k_{2}} U\left(\frac{\left|x_{i}-x_{j}\right|}{\varepsilon}\right) \\
& \quad+\varepsilon^{N-m+1} O\left(\sum_{i=1}^{k_{1}} e^{-(2+\sigma) d_{i} / \varepsilon}+\sum_{i=1}^{k_{1}} \sum_{j=k_{1}+1}^{k_{1}+k_{2}} e^{-(1+\sigma)\left|x_{i}-x_{j}\right| / \varepsilon}+\varepsilon\right) .
\end{aligned}
$$


Employing similar arguments as in [10], we conclude that

$$
\begin{aligned}
I_{\varepsilon}\left(\sum_{j=1}^{k_{1}+k_{2}} P_{\varepsilon, \Omega} W_{\varepsilon, x_{j}}\right) & =A \varepsilon^{N-m+1} \sum_{j=1}^{k_{1}} x_{j, 1}^{m-1}+\frac{1}{2} A \varepsilon^{N-m+1} \sum_{j=k_{1}+1}^{k_{1}+k_{2}} x_{j, 1}^{m-1} \\
& +\frac{1}{2} \sum_{j=1}^{k_{1}} \tau_{\varepsilon, x_{j}}-c_{0} \varepsilon^{N-m+1} \sum_{1 \leq i<j \leq k_{1}+k_{2}} U\left(\frac{\left|x_{i}-x_{j}\right|}{\varepsilon}\right) \\
& +\varepsilon^{N-m+1} O\left(\sum_{j=1}^{k_{1}} e^{-(2+\sigma) d_{j} / \varepsilon}+\sum_{j \neq i} e^{-(1+\sigma)\left|x_{i}-x_{j}\right| / \varepsilon}+\varepsilon\right),
\end{aligned}
$$

and $\tau_{\varepsilon, x_{j}}$ satisfies $(2.4)$.

\section{Proof of the main result}

Let

$$
\begin{aligned}
D_{\varepsilon}^{*}=\left\{x=\left(x_{1}, \ldots, x_{k_{1}+k_{2}}\right):\right. & x_{i} \in D, e^{-2 d_{i} / \varepsilon} \leq \varepsilon^{1-\tilde{\theta}}, i=1, \ldots, k_{1} \\
& x_{j} \in \partial D, j=k_{1}+1, \ldots, k_{1}+k_{2} ; \\
& x_{i} \in B_{\delta}(\bar{x}), e^{-\left|x_{i}-x_{j}\right| / \varepsilon} \leq \varepsilon^{1-\tilde{\theta}} \\
& \left.i, j=1, \cdots, k_{1}+k_{2}, i \neq j\right\},
\end{aligned}
$$

where $\tilde{\theta}>0$ is a fixed small constant, $d_{i}=d\left(x_{i}, \partial D\right)$.

Define

$$
J(x, \omega)=I_{\varepsilon}\left(\sum_{j=1}^{k_{1}+k_{2}} P_{\varepsilon, \Omega} W_{\varepsilon, x_{j}}+\omega\right), \quad \forall x \in D_{\varepsilon}^{*}, \omega \in H_{s} .
$$

Let

$$
\begin{gathered}
E_{\varepsilon, x, k_{1}+k_{2}}=\left\{\omega \in H_{s}:\left\langle\omega, \frac{\partial P_{\varepsilon, \Omega} W_{\varepsilon, x_{j}}}{\partial x_{j, l}}\right\rangle_{D, \varepsilon}=0,\right. \\
j=1, \cdots, k_{1}, l=1 \cdots, N-m+1 ; \\
\left\langle\omega, \frac{\partial P_{\varepsilon, \Omega} W_{\varepsilon, x_{j}}}{\partial \tau_{j, l}}\right\rangle_{D, \varepsilon}=0, \\
\left.j=k_{1}+1, \cdots, k_{1}+k_{2}, l=1, \cdots, N-m\right\},
\end{gathered}
$$

where

$$
\langle u, v\rangle_{D, \varepsilon}=\int_{D} z_{1}^{m-1}\left(\varepsilon^{2} D u D v+u v\right) d z,
$$

$\tau_{j, l}(l=1, \ldots, N-m)$ denotes the $N-1$ tangent vectors of $\partial \Omega$ at $x_{j} \in \partial \Omega$, $\left(j=k_{1}+1, \ldots, k_{1}+k_{2}\right)$. 
Lemma 3.1. There exists $\delta_{0}>0$ and $\varepsilon_{0}>0$ such that if $\varepsilon \in\left(0, \varepsilon_{0}\right]$ and $\delta \in\left(0, \delta_{0}\right],(x, \omega)$ is a critical point of $J$ in $D_{\varepsilon}^{*} \times E_{\varepsilon, x, k_{1}+k_{2}}$ if and only if

$$
u=\sum_{j=1}^{k_{1}+k_{2}} P_{\varepsilon, \Omega} W_{\varepsilon, x_{j}}+\omega_{\varepsilon}
$$

is a critical point of $I_{\varepsilon}$ in $H_{s}$.

The proof of Lemma 3.1 can be completed with the same arguments as in [3], we omit it here.

We notice that $(x, \omega)$ is a critical point of $J$ in $D_{\varepsilon}^{*} \times E_{\varepsilon, x, k_{1}+k_{2}}$ if and only if there are scalars $A_{j, l} \in \mathbb{R}, j=1, \ldots, k_{1}+k_{2}, l=1, \ldots, N-m+1$, such that

$$
\begin{aligned}
\frac{\partial J}{\partial x_{j, l}}= & \sum_{h=1}^{N-m+1} A_{j, h}\left\langle\frac{\partial^{2} P_{\varepsilon, \Omega} W_{\varepsilon, x_{j}}}{\partial x_{j, h} \partial x_{j, l}}, \omega\right\rangle_{D, \varepsilon}, \\
& j=1, \ldots, k_{1}, l=1, \ldots, N-m+1, \\
\frac{\partial J}{\partial \tau_{j, l}} & =\sum_{h=1}^{N-m} A_{j, h}\left\langle\frac{\partial^{2} P_{\varepsilon, \Omega} W_{\varepsilon, x_{j}}}{\partial \tau_{j, h} \partial \tau_{j, l}}, \omega\right\rangle_{D, \varepsilon}, \\
& j=k_{1}, \ldots, k_{1}+k_{2}, l=1, \ldots, N-m, \\
\frac{\partial J}{\partial w} & =\sum_{j=1}^{k_{1}} \sum_{l=1}^{N-m+1} A_{j, l} \frac{\partial P_{\varepsilon, \Omega} W_{\varepsilon, x_{j}}}{\partial x_{j, l}}+\sum_{j=k_{1}+1}^{k_{1}+k_{2}} \sum_{l=1}^{N-m} A_{j, l} \frac{\partial P_{\varepsilon, \Omega} W_{\varepsilon, x_{j}}}{\partial \tau_{j, l}} .
\end{aligned}
$$

In order to prove Theorem 1.1, we show first that for $x=\left(x_{1}, \ldots, x_{k_{1}+k_{2}}\right) \in D_{\varepsilon}^{*}$ given, $\varepsilon$ small enough, there exist $\omega_{\varepsilon, x} \in E_{\varepsilon, x, k_{1}+k_{2}}$ and scalars $A_{j, l}, j=$ $1, \ldots, k_{1}+k_{2}, l=1, \ldots, N-m+1$, such that (3.4) is satisfied and the mapping $x \rightarrow \omega_{\varepsilon, x}$ is $C^{1}$. We then show that for sufficiently small $\varepsilon$, there exists a point $x_{\varepsilon} \in D_{\varepsilon}^{*}$, such that $\left(x_{\varepsilon}, \omega\right) \in D_{\varepsilon}^{*} \times E_{\varepsilon, x, k_{1}+k_{2}}$ and (3.2), (3.3) are satisfied with these scalars $A_{j, l}$.

We expand $J(x, \omega)$ near $\omega=0$ as follows:

$$
J(x, \omega)=J(x, 0)+h_{\varepsilon, x}(\omega)+\frac{1}{2} Q_{\varepsilon, x}(\omega)-R_{\varepsilon, x}(\omega),
$$

where

$$
\begin{aligned}
h_{\varepsilon, x}(\omega)= & \sum_{j=1}^{k_{1}+k_{2}} \int_{\Omega}\left(\varepsilon^{2} D P_{\varepsilon, \Omega} W_{\varepsilon, x_{j}} D \omega+P_{\varepsilon, \Omega} W_{\varepsilon, x_{j}} \omega\right) \\
& -\int_{\Omega}\left(\sum_{j=1}^{k_{1}+k_{2}} P_{\varepsilon, \Omega} W_{\varepsilon, x_{j}}\right)_{+}^{p-1} \omega,
\end{aligned}
$$




$$
Q_{\varepsilon, x}(\omega)=\int_{\Omega}\left(\varepsilon^{2}|D \omega|^{2}+\omega^{2}\right)-(p-1) \int_{\Omega}\left(\sum_{j=1}^{k_{1}+k_{2}} P_{\varepsilon, \Omega} W_{\varepsilon, x_{j}}\right)_{+}^{p-2} \omega^{2}
$$

and

$$
R_{\varepsilon, x}(\omega)=\int_{\Omega} F\left(y, \sum_{j=1}^{k_{1}+k_{2}} P_{\varepsilon, \Omega} W_{\varepsilon, x_{j}}+\omega\right)-\int_{\Omega} F\left(y, \sum_{j=1}^{k_{1}+k_{2}} P_{\varepsilon, \Omega} W_{\varepsilon, x_{j}}\right)
$$

$$
-\int_{\Omega}\left(\sum_{j=1}^{k_{1}+k_{2}} P_{\varepsilon, \Omega} W_{\varepsilon, x_{j}}\right)_{+}^{p-1} \omega-\frac{1}{2}(p-1) \int_{\Omega}\left(\sum_{j=1}^{k_{1}+k_{2}} P_{\varepsilon, \Omega} W_{\varepsilon, x_{j}}\right)_{+}^{p-2} \omega^{2} .
$$

Lemma 3.2. There are constants $C>0$ and $\sigma>0$, such that

$$
\begin{aligned}
& \left|h_{\varepsilon, x}(\omega)\right| \\
& \quad \leq C \varepsilon^{(N-m+1) / 2}\left(\sum_{j=1}^{k_{1}} e^{-(1+\sigma) d_{j} / \varepsilon}+\sum_{j \neq i} e^{-(1+\sigma)\left|x_{i}-x_{j}\right| /(2 \varepsilon)}+\varepsilon^{(1+2 \sigma) / 2}\right)\|\omega\|_{\varepsilon} .
\end{aligned}
$$

Proof. We have

$$
\begin{aligned}
h_{\varepsilon, x}(\omega)= & \sum_{j=1}^{k_{1}+k_{2}} \int_{\Omega}\left(\xi \bar{W}_{\varepsilon, x_{j}}^{p-1}+\tilde{f}_{\varepsilon, x_{j}}(y)\right) \omega-\int_{\Omega}\left(\sum_{j=1}^{k_{1}+k_{2}} P_{\varepsilon, \Omega} W_{\varepsilon, x_{j}}\right)_{+}^{p-1} \omega \\
= & \sum_{j=1}^{k_{1}+k_{2}} \int_{\Omega}\left(\xi \bar{W}_{\varepsilon, x_{j}}^{p-1}-W_{\varepsilon, x_{j}}^{p-1}\right) \omega+\sum_{j=1}^{k_{1}+k_{2}} \int_{\Omega}\left(W_{\varepsilon, x_{j}}^{p-1}-\left(P_{\varepsilon, \Omega} W_{\varepsilon, x_{j}}\right)_{+}^{p-1}\right) \omega \\
& +\int_{\Omega}\left(\sum_{j=1}^{k_{1}+k_{2}}\left(P_{\varepsilon, \Omega} W_{\varepsilon, x_{j}}\right)_{+}^{p-1}-\left(\sum_{j=1}^{k_{1}+k_{2}} P_{\varepsilon, \Omega} W_{\varepsilon, x_{j}}\right)_{+}^{p-1}\right) \omega \\
& +\sum_{j=1}^{k_{1}+k_{2}} \int_{\Omega} \tilde{f}_{\varepsilon, x_{j}}(y) \omega \\
= & \left(\sum_{j=1}^{k_{1}+k_{2}} \int_{\Omega} W_{\varepsilon, x_{j}}^{(1+2 \sigma) / 2} \varphi_{\varepsilon, x_{j}}^{(1+2 \sigma) / 2}|\omega|\right)+O\left(e^{-\delta^{\prime} / \varepsilon}\right)\|\omega\|_{\varepsilon} \\
& +\sum_{j=1}^{k_{1}+k_{2}} \int_{\Omega} \tilde{f}_{\varepsilon, x_{j}}(y) \omega \\
& +O\left(\sum_{j \neq i} \int_{\Omega}\left|P_{\varepsilon, \Omega} W_{\varepsilon, x_{i}}\right|^{(p-1) / 2}\left|P_{\varepsilon, \Omega} W_{\varepsilon, x_{j}}\right|^{(p-1) / 2}|\omega|\right) .
\end{aligned}
$$


From Lemma 2.1 we obtain

$$
\begin{aligned}
\int_{\Omega} W_{\varepsilon, x_{j}}^{(1+2 \sigma) / 2} \varphi_{\varepsilon, x_{j}}^{(1+2 \sigma) / 2}|\omega| \leq\left(\int_{\Omega} W_{\varepsilon, x_{j}}^{(1+2 \sigma)} \varphi_{\varepsilon, x_{j}}^{(1+2 \sigma)}\right)^{1 / 2}\|\omega\|_{\varepsilon} \\
\quad \leq \begin{cases}C\left(e^{-(1+2 \sigma) d_{j} / \varepsilon-(1+2 \sigma)(1-\theta) d_{j} / \varepsilon} \int_{\Omega} W_{\varepsilon, x_{j}}^{(1+2 \sigma) \theta}\right)^{1 / 2}\|\omega\|_{\varepsilon} & \left(x_{j} \in D\right) \\
C \varepsilon^{(N-m+2+2 \sigma) / 2}\|\omega\|_{\varepsilon} & \left(x_{j} \in \partial D\right)\end{cases} \\
\quad \leq \begin{cases}C \varepsilon^{(N-m+1) / 2} e^{-(1+\sigma) d_{j} / \varepsilon}\|\omega\|_{\varepsilon} & \left(x_{j} \in D\right) \\
C \varepsilon^{(N-m+2+2 \sigma) / 2}\|\omega\|_{\varepsilon} & \left(x_{j} \in \partial D\right),\end{cases}
\end{aligned}
$$

where in the second inequality, we have used $\left|\tilde{y}-x_{j}\right|+d(\tilde{y}, \partial D) \geq d_{j}$ for $x_{j} \in D$.

Again by using Lemma 2.1, we deduce that for $i \neq j$,

$$
\int_{\Omega}\left|P_{\varepsilon, \Omega} W_{\varepsilon, x_{i}}\right|^{(1+2 \sigma) / 2}\left|P_{\varepsilon, \Omega} W_{\varepsilon, x_{j}}\right|^{(1+2 \sigma) / 2}|\omega|
$$$$
\leq\left(\int_{\Omega}\left|P_{\varepsilon, \Omega} W_{\varepsilon, x_{i}}\right|^{p-1}\left|P_{\varepsilon, \Omega} W_{\varepsilon, x_{j}}\right|^{p-1}\right)^{1 / 2}\|\omega\|_{\varepsilon}
$$$$
\leq\left\{\begin{array}{l}
C \varepsilon^{(N-m+1) / 2} e^{-(1+\sigma)\left|x_{i}-x_{j}\right| /(2 \varepsilon)}\|\omega\|_{\varepsilon}\left(x_{i}, x_{j} \in \Omega\right) \\
C\left(\varepsilon^{(N-m+1) / 2} e^{-(1+\sigma)\left|x_{i}-x_{j}\right| /(2 \varepsilon)}+\varepsilon^{(N-m+p) / 2}\right)\|\omega\|_{\varepsilon} \quad\left(x_{i} \in \Omega, x_{j} \in \partial \Omega\right) \\
C\left(\varepsilon^{(N-m+1) / 2} e^{-(1+\sigma)\left|x_{i}-x_{j}\right| /(2 \varepsilon)}+\varepsilon^{(N-m+2 p-1) / 2}\right)\|\omega\|_{\varepsilon} \quad\left(x_{i}, x_{j} \in \partial \Omega\right) .
\end{array}\right.
$$

On the other hand,

$$
\left|\int_{\Omega} \tilde{f}_{\varepsilon, x_{j}}(y) \omega\right| \leq\left(\int_{\Omega}\left|\tilde{f}_{\varepsilon, x_{j}}(y)\right|^{2}\right)^{1 / 2}\|\omega\|_{\varepsilon} \leq C \varepsilon^{1+(N-m+1) / 2}\|\omega\|_{\varepsilon} .
$$

Combining (3.8)-(3.11), we obtain the result.

Let $Q_{\varepsilon, x}$ be the bounded linear map $E_{\varepsilon, x, k_{1}+k_{2}}$ to $E_{\varepsilon, x, k_{1}+k_{2}}$, such that

$$
\left\langle Q_{\varepsilon, x} \omega_{1}, \omega_{2}\right\rangle_{\varepsilon}=\int_{\Omega}\left(\varepsilon^{2} D \omega_{1} D \omega_{2}+\omega_{1} \omega_{2}\right)-(p-1) \int_{\Omega}\left(\sum_{j=1}^{k_{1}+k_{2}} P_{\varepsilon, \Omega} W_{\varepsilon, x_{j}}\right)_{+}^{p-2} \omega_{1} \omega_{2},
$$

for $\omega_{1}, \omega_{2} \in E_{\varepsilon, x, k_{1}+k_{2}}$. Then we have

Lemma 3.3. There are constants $\varepsilon_{0}>0$ and $\rho>0$, such that for each $\varepsilon \in\left(0, \varepsilon_{0}\right]$, and $x \in D_{\varepsilon}^{*}$,

$$
\left\|Q_{\varepsilon, x} \omega\right\|_{\varepsilon} \geq \rho\|\omega\|_{\varepsilon}, \quad \omega \in E_{\varepsilon, x, k_{1}+k_{2}} .
$$


Proof. Suppose to the contrary that Lemma 3.3 does not hold, then there exist $\varepsilon_{n} \rightarrow 0, x_{n}=\left(x_{n, 1}, \ldots, x_{n, k_{1}+k_{2}}\right) \in D_{\varepsilon_{n}}^{*}$ and $\omega_{n} \in E_{\varepsilon_{n}, x_{n}, k_{1}+k_{2}}$, such that

$$
\left\|Q_{\varepsilon_{n}, x_{n}} \omega_{n}\right\|_{\varepsilon_{n}}=o_{n}(1)\left\|\omega_{n}\right\|_{\varepsilon_{n}}
$$

that is

$$
\begin{aligned}
& \int_{\Omega}\left(\varepsilon_{n}^{2} D \omega_{n} D \varphi+\omega_{n} \varphi\right)-(p-1) \int_{\Omega}\left(\sum_{j=1}^{k_{1}+k_{2}} P_{\varepsilon_{n}, \Omega} W_{\varepsilon_{n}, x_{n, j}}\right)_{+}^{p-2} \omega_{n} \varphi \\
&=o_{n}(1)\left\|\omega_{n}\right\|_{\varepsilon_{n}}\|\varphi\|_{\varepsilon_{n}}, \quad \forall \varphi \in E_{\varepsilon_{n}, x_{n}, k_{1}+k_{2}} .
\end{aligned}
$$

Assume without loss of generality that

$$
\left\|\omega_{n}\right\|_{\varepsilon_{n}}=\varepsilon_{n}^{\frac{N-m+1}{2}} .
$$

For each fixed $j \in\left\{1, \ldots, k_{1}+k_{2}\right\}$, let $\tilde{\omega}_{n, j}(\tilde{y})=\omega_{n}\left(\varepsilon_{n} \tilde{y}+x_{n, j}\right)$. Since $x_{n, j, 1}>c>0$, by $(3.12)$,

$$
\int_{B_{R}}\left(\left|D \tilde{\omega}_{n, j}\right|^{2}+\left|\tilde{\omega}_{n, j}\right|^{2}\right) \leq C
$$

for any $R>0$ large, where $C>0$ is in dependent of $R, B_{R}=B_{R}(0)$ for $j=1, \ldots, k_{1}$ and $B_{R}=B_{R}(0) \cap \mathbb{R}_{+}^{N-m+1}$ for $j=k_{1}+1, \ldots, k_{1}+k_{2}, B_{R}(0)$ is the ball in $\mathbb{R}^{N-m+1}$ with radius $R$ and centered at the origin.

Thus there is a subsequence (still denoted by $\{n\}$ ) and an $\omega \in H^{1}\left(\mathbb{R}^{N-m+1}\right)$, such that for any $R>0$,

$\tilde{\omega}_{n, j} \rightarrow \omega$, weakly in $H^{1}\left(B_{R}\right), \quad$ and $\quad \tilde{\omega}_{n, j} \rightarrow \omega$, strongly in $L^{2}\left(B_{R}\right)$.

Moreover, by the nondegeneracy properties of $U$ which were stated in Section 1 and using the similar arguments to $[10,6]$ (see also [3, 8]), we deduce that $\omega \equiv 0$.

Now, for $j=1, \ldots, k_{1}+k_{2}$, let $B_{j, R}=\left\{y \in \Omega:\left(\left|y^{\prime}\right|, y^{\prime \prime}\right) \in B_{\varepsilon R}\left(x_{n, j}\right) \cap D\right\}$. Then,

$$
\begin{aligned}
& \int_{\Omega}\left(\sum_{j=1}^{k_{1}+k_{2}} P_{\varepsilon_{n}, \Omega} W_{\varepsilon_{n}, x_{n, j}}\right)^{p-2} \omega_{n}^{2} d y \\
& =\int_{\cup_{j=1}^{k_{1}+k_{2}} B_{j, R}}\left(\sum_{j=1}^{k_{1}+k_{2}} P_{\varepsilon_{n}, \Omega} W_{\varepsilon_{n}, x_{n, j}}\right)^{p-2} \omega_{n}^{2} d y+\int_{\Omega \backslash \cup_{j=1}^{k_{1}+k_{2}} B_{j, R}}\left(\sum_{j=1}^{k_{1}+k_{2}} P_{\varepsilon_{n}, \Omega} W_{\varepsilon_{n}, x_{n, j}}\right)^{p-2} \omega_{n}^{2} d y \\
& \leq C \int_{\cup_{j=1}^{k_{1}+k_{2}} B_{j, R}} \omega_{n}^{2} d x+o_{R}(1)\left\|\omega_{n}\right\|_{\varepsilon_{n}}^{2}=o\left(\varepsilon_{n}^{N-m+1}\right)+o_{R}(1) \varepsilon_{n}^{N-m+1},
\end{aligned}
$$

where $o_{R}(1) \rightarrow 0$ as $R \rightarrow \infty$. 
Hence from (3.12), we have

$$
o\left(\varepsilon_{n}^{N-m+1}\right)=\left\|w_{n}\right\|_{\varepsilon_{n}}^{2}+o\left(\varepsilon_{n}^{N-m+1}\right)+o_{R}(1) \varepsilon_{n}^{N-m+1},
$$

which is impossible.

As a result, we complete the proof.

Let

$$
S_{\varepsilon}=\left\{\omega: \omega \in H_{s}(\Omega),|\omega| \leq \sum_{j=1}^{k_{1}+k_{2}} e^{-\alpha\left|\tilde{y}-x_{j}\right| / \varepsilon}\right\},
$$

where $\alpha>0$ is a small constant.

Lemma 3.4. For any $\omega \in S_{\varepsilon}$ with $\|\omega\|_{\varepsilon} \leq \varepsilon^{(N-m+1) / 2}$, we have

$$
\begin{aligned}
R_{\varepsilon, x}(\omega) & =\varepsilon^{N-m+1} O\left(\varepsilon^{-p^{*}(N-m+1) / 2}\|\omega\|_{\varepsilon}^{p^{*}}\right), \\
\left\langle R_{\varepsilon, x}^{\prime}(\omega), \xi\right\rangle_{\varepsilon} & =\varepsilon^{(N-m+1) / 2} O\left(\varepsilon^{-\left(p^{*}-1\right)(N-m+1) / 2}\|\omega\|_{\varepsilon}^{p^{*}-1}\right)\|\xi\|_{\varepsilon}
\end{aligned}
$$

and

$$
R_{\varepsilon, x}^{\prime \prime}(\omega)\left(\xi_{1}, \xi_{2}\right)=O\left(\varepsilon^{-\left(p^{*}-2\right)(N-m+1) / 2}\|\omega\|_{\varepsilon}^{p^{*}-2}\right)\left\|\xi_{1}\right\|_{\varepsilon}\left\|\xi_{2}\right\|_{\varepsilon}
$$

where $p^{*}=\min \{p, 3\}$.

Proof. The proof of this lemma is similar to the proof of Lemma 3.3 in [10]. Thus, we omit it.

Proposition 3.1. There is an $\varepsilon_{0}>0$, such that for each $\varepsilon \in\left(0, \varepsilon_{0}\right]$, there exists a $C^{1}$-map $\omega_{\varepsilon, x}: D_{\varepsilon}^{*} \rightarrow H_{s}$ such that $\omega_{\varepsilon, x} \in E_{\varepsilon, x, k_{1}+k_{2}}$, (3.4) holds for some constants $A_{j l}$. Moreover, we have

$$
\left\|\omega_{\varepsilon, x}\right\|_{\varepsilon} \leq C \varepsilon^{\sigma+(N-m+2) / 2},
$$

where $\sigma>0$ is a constant.

Proof. By Lemma 3.2, we know that there is a $h_{\varepsilon, x} \in E_{\varepsilon, x, k_{1}+k_{2}}$, such that

$$
\left\langle h_{\varepsilon, x}, \omega\right\rangle_{\varepsilon}=h_{\varepsilon, x}(\omega), \quad \forall \omega \in E_{\varepsilon, x, k_{1}+k_{2}} .
$$

Thus, solving (3.17) is equivalent to solving

$$
h_{\varepsilon, x}+Q_{\varepsilon, x} \omega+R_{\varepsilon, x}^{\prime}(\omega)=0, \quad \text { in } E_{\varepsilon, x, k_{1}+k_{2}} .
$$

By Lemma $3.3, Q_{\varepsilon, x}$ is invertible. So we can write (3.18) as

$$
\omega=G_{\varepsilon, x} \omega=:-Q_{\varepsilon, x}^{-1} h_{\varepsilon, x}-Q_{\varepsilon, x}^{-1} R_{\varepsilon, x}^{\prime}(\omega) .
$$


Let

$$
\tilde{S}_{\varepsilon}=\left\{\omega: \omega \in H_{s}(\Omega),|\omega| \leq \varepsilon^{\alpha} \sum_{j=1}^{k_{1}+k_{2}} e^{-\alpha\left|\tilde{y}-x_{j}\right| / \varepsilon},\|\omega\|_{\varepsilon} \leq \varepsilon^{(N-m+2) / 2}\right\},
$$

where $\alpha>0$ is a small constant.

Now, we prove that $G_{\varepsilon, x}$ is a contraction map from $\tilde{S}_{s}$ to $\tilde{S}_{\varepsilon}$.

By (3.16), we see that for any $\omega_{1}, \omega_{2} \in \tilde{S}_{\varepsilon}$,

$$
\left\|G_{\varepsilon, x} \omega_{1}-G_{\varepsilon, x} \omega_{2}\right\|_{\varepsilon} \leq C\left\|R_{\varepsilon, x}^{\prime}\left(\omega_{1}\right)-R_{\varepsilon, x}^{\prime}\left(\omega_{2}\right)\right\|_{\varepsilon} \leq C \varepsilon^{\left(p^{*}-2\right) / 2}\left\|\omega_{1}-\omega_{2}\right\|_{\varepsilon} .
$$

Thus, $G_{\varepsilon, x}$ is a contraction map. Moreover, by Lemma 3.2 and (3.15),

$$
\left\|G_{\varepsilon, x} \omega\right\|_{\varepsilon} \leq C\left\|h_{\varepsilon, x}\right\|_{\varepsilon}+C\left\|R_{\varepsilon, x}^{\prime}(\omega)\right\|_{\varepsilon} \leq C \varepsilon^{\sigma_{1}+(N-m+2) / 2} \leq \varepsilon^{(N-m+2) / 2} .
$$

To finish the proof of $G_{\varepsilon, x} \omega \in \tilde{S}_{\varepsilon}$, we need to prove

$$
\left|G_{\varepsilon, x} \omega\right| \leq \varepsilon^{\alpha} \sum_{j=1}^{k_{1}+k_{2}} e^{-\alpha\left|\tilde{y}-x_{j}\right| / \varepsilon}
$$

Let $\omega_{1}=G_{\varepsilon, x} \omega$. Then, we have

$$
Q_{\varepsilon, x} \omega_{1}=-h_{\varepsilon, x}-R^{\prime}(\omega), \quad \text { in } E_{\varepsilon, x, k},
$$

which is equivalent to

$Q_{\varepsilon, x} \omega_{1}+h_{\varepsilon, x}+R^{\prime}(\omega)=\sum_{j=1}^{k_{1}} \sum_{h=1}^{N-m+1} A_{j h} \frac{\partial P_{\varepsilon, \Omega} W_{\varepsilon, x_{j}}}{\partial x_{j, h}}+\sum_{j=k_{1}+1}^{k_{1}+k_{2}} \sum_{h=1}^{N-m} A_{j h} \frac{\partial P_{\varepsilon, \Omega} W_{\varepsilon, x_{j}}}{\partial \tau_{j, h}}$

for some $A_{j h} \in \mathbb{R}$.

We claim that there is a $\sigma>0$, such that

$$
\left|A_{j h}\right| \leq C \varepsilon^{\sigma+3 / 2}, \quad j=1, \cdots, k, h=1, \cdots, N-m+1 .
$$

In fact, taking the scalar product in $H^{s}$ of (3.22) with

$$
\begin{aligned}
\frac{\partial P_{\varepsilon, \Omega} W_{\varepsilon, x_{j}}}{\partial x_{j, l}} & j=1, \ldots, k_{1}, \quad l=1, \ldots, N-m+1 \\
\text { and } \frac{\partial P_{\varepsilon, \Omega} W_{\varepsilon, x_{j}}}{\partial \tau_{j, l}} & j=k_{1}, \ldots, k_{1}+k_{2}, \quad l=1, \ldots, N-m
\end{aligned}
$$

respectively, we get a quasi-diagonal linear system with $A_{j, l}$ as unknown. Obviously, by Lemma 4.1 in Appendix, there exists $\varepsilon^{*}>0$, such that if $\varepsilon<\varepsilon^{*}$, the coefficient matrix of this linear system is invertible, which means

$$
\begin{aligned}
\left|A_{j h}\right| & \leq C \varepsilon^{1-(N-m+1) / 2}\left(\left\|\omega_{1}\right\|_{\varepsilon}+\left\|h_{\varepsilon, x}\right\|_{\varepsilon}+\left\|R^{\prime}(\omega)\right\|_{\varepsilon}\right) \\
& \leq C \varepsilon^{1-(N-m+1) / 2} \varepsilon^{\frac{1}{2}+\sigma+(N-m+1) / 2} \leq C \varepsilon^{\sigma+3 / 2} .
\end{aligned}
$$


Rewrite (3.22) as

$$
\begin{aligned}
-\varepsilon^{2} \Delta \omega_{1} & +\omega_{1}-(p-1)\left(\sum_{j=1}^{k_{1}+k_{2}} P_{\varepsilon, \Omega} W_{\varepsilon, x_{j}}\right)_{+}^{p-2} \omega_{1} \\
= & -\sum_{j=1}^{k_{1}+k_{2}}\left(\xi \bar{W}_{\varepsilon, x_{j}}^{p-1}+\tilde{f}_{\varepsilon, x_{j}}(y)\right)+\left(\sum_{j=1}^{k_{1}+k_{2}} P_{\varepsilon, \Omega} W_{\varepsilon, x_{j}}\right)_{+}^{p-1} \\
& -\left(f\left(y, \sum_{j=1}^{k_{1}+k_{2}} P_{\varepsilon, \Omega} W_{\varepsilon, x_{j}}+\omega\right)-f\left(y, \sum_{j=1}^{k_{1}+k_{2}} P_{\varepsilon, \Omega} W_{\varepsilon, x_{j}}\right)\right. \\
& \left.-f^{\prime}\left(y, \sum_{j=1}^{k_{1}+k_{2}} P_{\varepsilon, \Omega} W_{\varepsilon, x_{j}}\right) \omega\right) \\
& +\sum_{j=1}^{k_{1}} \sum_{h=1}^{N-m+1} A_{j h} \frac{\partial g_{\varepsilon, x_{j}}(y)}{\partial x_{j, h}}+\sum_{j=k_{1}+1}^{k_{1}+k_{2}} \sum_{h=1}^{N-m} A_{j h} \frac{\partial g_{\varepsilon, x_{j}}(y)}{\partial \tau_{j, h}}=: G_{\varepsilon, x}(y),
\end{aligned}
$$

where $f(y, t)$ is the function defined in (1.6), and $g_{\varepsilon, x_{j}}(y)=\xi W_{\varepsilon, x_{j}}^{p-1}+\tilde{f}_{\varepsilon, x_{j}}(y)$.

Since $\omega \in \tilde{S}_{\varepsilon}$, we see $|\omega| \leq \frac{1}{2}$ in $\Omega \backslash B$. Thus

$$
\begin{aligned}
\mid f\left(y, \sum_{j=1}^{k_{1}+k_{2}} P_{\varepsilon, \Omega} W_{\varepsilon, x_{j}}+\omega\right)- & f\left(y, \sum_{j=1}^{k_{1}+k_{2}} P_{\varepsilon, \Omega} W_{\varepsilon, x_{j}}\right) \\
& -\left.f^{\prime}\left(y, \sum_{j=1}^{k_{1}+k_{2}} P_{\varepsilon, \Omega} W_{\varepsilon, x_{j}}\right)|\leq C| \omega\right|^{p^{*}-1} .
\end{aligned}
$$

Direct calculations lead to

$$
\begin{aligned}
-\sum_{j=1}^{k_{1}+k_{2}} \xi \bar{W}_{\varepsilon, x_{j}}^{p-1}+\left(\sum_{j=1}^{k_{1}+k_{2}} P_{\varepsilon, \Omega} W_{\varepsilon, x_{j}}\right)_{+}^{p-1} & \\
=- & \sum_{j=1}^{k_{1}+k_{2}}\left(W_{\varepsilon, x_{j}}^{p-1}-\left(P_{\varepsilon, \Omega} W_{\varepsilon, x_{j}}\right)_{+}^{p-1}\right)+\sum_{j=1}^{k_{1}+k_{2}} O\left(e^{-\delta^{\prime} / \varepsilon} W_{\varepsilon, x_{j}}\right) \\
& +\left(\sum_{j=1}^{k_{1}+k_{2}} P_{\varepsilon, \Omega} W_{\varepsilon, x_{j}}\right)_{+}^{p-1}-\sum_{j=1}^{k_{1}+k_{2}}\left(P_{\varepsilon, \Omega} W_{\varepsilon, x_{j}}\right)_{+}^{p-1} \\
= & O\left(\sum_{j=1}^{k} e^{-d_{j} / \varepsilon} U^{p-2}\left(\frac{\left|\tilde{y}-x_{j}\right|}{\varepsilon}\right)+\sum_{j=k_{1}+1}^{k_{1}+k_{2}} \varepsilon U^{p-2}\left(\frac{\left|\tilde{y}-x_{j}\right|}{\varepsilon}\right)\right. \\
& \left.+\sum_{j=1}^{k_{1}+k_{2}} e^{-\delta^{\prime} / \varepsilon} W_{\varepsilon, x_{j}}+\sum_{i \neq j}\left|P_{\varepsilon, \Omega} W_{\varepsilon, x_{i}}\right|^{(1+\sigma) / 2}\left|P_{\varepsilon, \Omega} W_{\varepsilon, x_{j}}\right|^{(1+\sigma) / 2}\right) .
\end{aligned}
$$


But by Lemma 2.1, we see for $x_{i} \in \bar{D}$

$$
\left|P_{\varepsilon, \Omega} W_{\varepsilon, x_{i}}\right| \leq C e^{-(1-\theta)\left|\tilde{y}-x_{j}\right|},
$$

so, we deduce that

$$
\begin{aligned}
& \left|P_{\varepsilon, \Omega} W_{\varepsilon, x_{i}}\right|^{(1+\sigma) / 2}\left|P_{\varepsilon, \Omega} W_{\varepsilon, x_{j}}\right|^{(1+\sigma) / 2} \\
& \quad \leq C e^{-(1+\sigma)(1-\theta)\left|x_{i}-x_{j}\right| /(4 \varepsilon)} e^{-(1-\theta)(1+\sigma)\left|\tilde{y}-x_{i}\right| /(4 \varepsilon)} e^{-(1-\theta)(1+\sigma)\left|\tilde{y}-x_{j}\right| /(4 \varepsilon)} \\
& \quad \leq C e^{-\left(1+\sigma^{\prime}\right)\left|x_{i}-x_{j}\right| /(4 \varepsilon)}\left(e^{-\left(1+\sigma^{\prime}\right)\left|\tilde{y}-x_{i}\right| /(2 \varepsilon)}+e^{-\left(1+\sigma^{\prime}\right)\left|\tilde{y}-x_{j}\right| /(2 \varepsilon)}\right),
\end{aligned}
$$

for some $\sigma^{\prime}>0$. Combining (3.26) and (3.27), we are led to

$$
\begin{aligned}
& -\sum_{j=1}^{k_{1}+k_{2}} \xi \bar{W}_{\varepsilon, x_{j}}^{p-1}+\left(\sum_{j=1}^{k_{1}+k_{2}} P_{\varepsilon, \Omega} W_{\varepsilon, x_{j}}\right)_{+}^{p-1} \\
\leq & C\left(\sum_{j=1}^{k_{1}} e^{-d_{j} / \varepsilon} U^{p-2}\left(\frac{\left|\tilde{y}-x_{j}\right|}{\varepsilon}\right)\right. \\
& \left.+\sum_{j=k_{1}+1}^{k_{1}+k_{2}} \varepsilon U^{p-2}\left(\frac{\left|\tilde{y}-x_{j}\right|}{\varepsilon}\right)+\sum_{j=1}^{k_{1}+k_{2}} e^{-\delta^{\prime} / \varepsilon} W_{\varepsilon, x_{j}}\right) \\
& +C \sum_{i \neq j} e^{-\left(1+\sigma^{\prime}\right)\left|x_{i}-x_{j}\right| /(4 \varepsilon)}\left(e^{-\left(1+\sigma^{\prime}\right)\left|\tilde{y}-x_{i}\right| /(2 \varepsilon)}+e^{-\left(1+\sigma^{\prime}\right)\left|\tilde{y}-x_{j}\right| /(4 \varepsilon)}\right) .
\end{aligned}
$$

Direct calculations show that for $x_{i} \in D, h=1, \ldots, N-m+1$ and $x_{j} \in \partial D, l=1, \ldots, N-m$

$$
\begin{aligned}
& \left|\frac{\partial g_{\varepsilon, x_{i}}(y)}{\partial x_{i, h}}\right| \leq C \varepsilon^{-1} U^{p-1}\left(\frac{\left|\tilde{y}-x_{i}\right|}{\varepsilon}\right)+C U\left(\frac{\left|\tilde{y}-x_{i}\right|}{\varepsilon}\right), \\
& \left|\frac{\partial g_{\varepsilon, x_{j}}(y)}{\partial \tau_{j, l}}\right| \leq C \varepsilon^{-1} U^{p-1}\left(\frac{\left|\tilde{y}-x_{j}\right|}{\varepsilon}\right)+C U\left(\frac{\left|\tilde{y}-x_{j}\right|}{\varepsilon}\right) .
\end{aligned}
$$

Combining (3.23), (3.25), (3.28), (3.29) and (3.30), we find that

$\left|G_{\varepsilon, x}(y)\right| \leq C \sum_{j=1}^{k_{1}+k_{2}}\left(e^{-\delta^{\prime} / \varepsilon} U\left(\frac{\left|\tilde{y}-x_{j}\right|}{\varepsilon}\right)+\varepsilon^{\sigma+1 / 2} U\left(\frac{\left|\tilde{y}-x_{j}\right|}{\varepsilon}\right)\right)+|\omega|^{p^{*}-1}$

$$
\begin{aligned}
& +C \sum_{j=1}^{k_{1}} e^{-d_{j} / \varepsilon} U^{p-2}\left(\frac{\left|\tilde{y}-x_{j}\right|}{\varepsilon}\right)+C \sum_{j=k_{1}+1}^{k_{1}+k_{2}} \varepsilon U^{p-2}\left(\frac{\left|\tilde{y}-x_{j}\right|}{\varepsilon}\right) \\
& +C \sum_{i \neq j} e^{-\left(1+\sigma^{\prime}\right)\left|x_{i}-x_{j}\right| /(4 \varepsilon)}\left(e^{-\left(1+\sigma^{\prime}\right)\left|\tilde{y}-x_{i}\right| /(2 \varepsilon)}+e^{-\left(1+\sigma^{\prime}\right)\left|\tilde{y}-x_{j}\right| /(4 \varepsilon)}\right) \\
\leq C & \sum_{j=1}^{k_{1}+k_{2}}\left(\varepsilon^{\alpha} e^{-\alpha\left|\tilde{y}-x_{j}\right| / \varepsilon}\right)^{p^{*}-1} .
\end{aligned}
$$

if $\alpha>0$ is small enough. 
With the same technique in [10] and using the theory of $L^{p}$-estimate and Schauder estimate on elliptic equation, (3.31) yields

$$
\left|\omega_{1}\right| \leq C \varepsilon^{\left(p^{*}-1\right) \alpha}, \quad \text { in } B \text {. }
$$

Let

$$
a_{\varepsilon}(y)=\left(\sum_{j=1}^{k_{1}+k_{2}} P_{\varepsilon, \Omega} W_{\varepsilon, x_{j}}\right)^{p-2} \eta,
$$

where $\eta$ is a $C^{1}$ function, such that $\eta=0$ if $y \in B$. It is easy to see that $a_{\varepsilon}(y) \rightarrow 0$ uniformly in $\Omega$ as $\varepsilon \rightarrow 0$. From (3.32), we have

$$
-\varepsilon^{2} \Delta \omega_{1}+\left(1-(p-1) a_{\varepsilon}\right) \omega_{1}=G_{\varepsilon, x}(y)+O\left(\varepsilon^{(\tilde{p}-1) \alpha}\right)\left(\sum_{j=1}^{k_{1}+k_{2}} W_{\varepsilon, x_{j}}\right)^{p-2}
$$

Let $\tilde{\omega} \in H$ be the solution of

$$
\left\{\begin{aligned}
-\varepsilon^{2} \Delta \tilde{\omega}+\frac{1}{2} \tilde{\omega} & =\left|G_{\varepsilon, x}(y)+O\left(\varepsilon^{(\tilde{p}-1) \alpha}\right)\left(\sum_{j=1}^{k_{1}+k_{2}} W_{\varepsilon, x_{j}}\right)^{p-2}\right|, & & \text { in } \Omega \\
\frac{\partial \tilde{\omega}}{\partial n} & =0, & & \text { on } \partial \Omega .
\end{aligned}\right.
$$

By the maximum principle, we have $\tilde{\omega}>0$.

Let $v=\omega_{1}-\tilde{\omega}$. Then

$$
-\varepsilon^{2} \Delta v+\frac{1}{2} v \leq-\left(\frac{1}{2}-(p-1) a_{\varepsilon}\right) \omega_{1} .
$$

Multiplying the above relation by $v_{+}$and integrating by part, we obtain

$$
\int_{\Omega}\left(\varepsilon^{2}\left|D v_{+}\right|^{2}+\frac{1}{2} v_{+}^{2}\right) \leq-\left(\frac{1}{2}-(p-1) a_{\varepsilon}\right) \int_{\Omega} v_{+} \omega_{1} \leq 0 .
$$

Thus, $v_{+}=0$. That is, $\omega_{1} \leq \tilde{\omega}$. Similarly, $-\omega_{1} \leq \tilde{\omega}$. Hence,

$$
\left|\omega_{1}\right| \leq \tilde{\omega}
$$

Now, by the estimate (3.31) and the arguments used in the proof of Lemma 2.1 , it is easy to verify

$$
\left|\omega_{1}(y)\right| \leq \tilde{\omega}(y) \leq \varepsilon^{\alpha} \sum_{j=1}^{k_{1}+k_{2}} e^{-\alpha\left|y-x_{j}\right| / \varepsilon} .
$$

Therefore, the contraction mapping theorem yields that there exists an $\omega_{\varepsilon, x} \in \tilde{S}_{\varepsilon, x}$, such that

Moreover, by (3.21),

$$
\omega_{\varepsilon, x}=G_{\varepsilon, x} \omega_{\varepsilon, x} .
$$

$$
\left\|\omega_{\varepsilon, x}\right\|_{\varepsilon} \leq C \varepsilon^{\sigma+(N-m+2) / 2} .
$$


In the following, we will choose $x_{\varepsilon} \in D_{\varepsilon}^{*}$, such that (3.2) and (3.3) are satisfied with $A_{j, l}$.

Proof of Theorem 1.1. By our assumption on $\Omega$, we can deduce that there is a constant $\delta^{\prime} \in(0, \delta)$, such that

$$
\max _{z^{\prime \prime} \in B_{\delta}(\bar{x}) \backslash B_{\delta^{\prime}}(\bar{x})} \psi\left(z^{\prime \prime}\right)<\max _{z^{\prime \prime} \in B_{\delta}(\bar{x})} \psi\left(z^{\prime \prime}\right) .
$$

Define

$$
D^{*}=\left\{z=\left(z_{1}, z^{\prime \prime}\right): z_{1} \in\left(\psi\left(z^{\prime \prime}\right)-\gamma, \psi\left(z^{\prime \prime}\right)\right), z^{\prime \prime} \in B_{\delta^{\prime}}(\bar{x})\right\}
$$

where $\gamma>0$ is a small constant.

Define

$$
D_{\varepsilon}=\left\{x=\left(x_{1}, \cdots, x_{k_{1}+k_{2}}\right): x \in D_{\varepsilon}^{*}, x_{j} \in D^{*}, j=1, \cdots, k_{1}\right\} .
$$

Let

$$
K(x)=J\left(x, \omega_{\varepsilon, x}\right), \quad x \in D_{\varepsilon} .
$$

Consider the following problem:

$$
\max _{x \in \bar{D}_{\varepsilon}} K(x)
$$

Let $x_{\varepsilon} \in \bar{D}_{\varepsilon}$ be a maximum point of (3.38). We will prove that $x_{\varepsilon}$ is an interior point of $D_{\varepsilon}$. Thus, $x_{\varepsilon}$ is a critical point of $K(x)$.

It follows from Propositions 3.1 and 2.1 that for any $x \in D_{\varepsilon}$,

$$
\begin{aligned}
K(x)= & J(x, 0)+O\left(\varepsilon^{N-m+2+\sigma}\right) \\
= & A \varepsilon^{N-m+1} \sum_{j=1}^{k_{1}} x_{j, 1}^{m-1}+\frac{1}{2} A \varepsilon^{N-m+1} \sum_{j=k_{1}+1}^{k_{1}+k_{2}} x_{j, 1}^{m-1} \\
& +\frac{1}{2} \sum_{j=1}^{k_{1}} \tau_{\varepsilon, x_{j}}-c_{0} \varepsilon^{N-m+1} \sum_{i<j} U\left(\frac{\left|x_{i}-x_{j}\right|}{\varepsilon}\right) \\
& +\varepsilon^{N-m+1} O\left(\sum_{j=1}^{k_{1}} e^{-(2+\sigma) d_{j} / \varepsilon}+\sum_{j \neq i} e^{-(1+\sigma)\left|x_{i}-x_{j}\right| / \varepsilon}+\varepsilon\right) .
\end{aligned}
$$

Let $\bar{x}_{\varepsilon, j}=\left(\bar{x}_{\varepsilon, j, 1}, x_{\varepsilon, j}^{\prime \prime}\right) \in D, \bar{x}_{\varepsilon, j, 1}=\bar{x}_{1}-\operatorname{Lj} \varepsilon|\ln \varepsilon| \psi\left(\bar{x}^{\prime \prime}\right), \bar{x}_{\varepsilon, j}^{\prime \prime}=\bar{x}^{\prime \prime}$, $\bar{x}_{\varepsilon}=\left(\bar{x}_{\varepsilon, 1}, \cdots, \bar{x}_{\varepsilon, k}\right), j=1, \ldots, k_{1}$, and $\bar{x}_{\varepsilon, j}=\left(\bar{x}_{\varepsilon, j, 1}, x_{\varepsilon, j}^{\prime \prime}\right) \in \partial D, \bar{x}_{\varepsilon, j, 1}=$ $\bar{x}_{1}-L j \varepsilon|\ln \varepsilon|, j=k_{1}, \ldots, k_{1}+k_{2}$, where $L>0$ are large constants. Then $\bar{x}_{\varepsilon} \in D_{\varepsilon}$. Moreover,

$$
\tau_{\varepsilon, \bar{x}_{j}}=O\left(\varepsilon^{N-m+3}\right)
$$


for $L>0$ large. It is easy to see that for $j \neq i$,

$$
e^{-\left|x_{i}-x_{j}\right| / \varepsilon}=O\left(\varepsilon^{N-m+3}\right) .
$$

So, from (3.39), we obtain

$$
K\left(\bar{x}_{\varepsilon}\right)=A\left(k_{1}+k_{2} / 2\right) \varepsilon^{N-m+1} \bar{x}_{1}^{m-1}+\varepsilon^{N-m+1} O(\varepsilon|\ln \varepsilon|) .
$$

Note that for any $x \in D_{\varepsilon}$, we have

$$
\tau_{\varepsilon, x_{j}}<0, j=1, \ldots, k_{1} ; \quad x_{j, 1} \leq \bar{x}_{1}, j=1, \ldots, k_{1}+k_{2} .
$$

Suppose that $x_{\varepsilon} \in \partial D_{\varepsilon}$.

If $e^{-2 d\left(x_{\varepsilon, j}, \partial D\right) / \varepsilon}=\varepsilon^{1-\tilde{\theta}}$ for some $j \in\left\{1, \ldots, k_{1}\right\}$, then, by (3.39),

$$
\begin{aligned}
K\left(x_{\varepsilon}\right) & \leq A\left(k_{1}+k_{2} / 2\right) \varepsilon^{N-m+1} \bar{x}_{1}^{m-1}-\frac{1}{2} \varepsilon^{N-m+1+(1+\theta)(1-\tilde{\theta})}+O\left(\varepsilon^{N-m+2}\right) \\
& <K\left(\bar{x}_{\varepsilon}\right)
\end{aligned}
$$

since $(1-\tilde{\theta})(1+\theta)<1$ if $\theta>0$ is small enough. This is a contradiction.

Suppose that there is a $j \in\left\{1, \ldots, k_{1}\right\}$, such that $x_{\varepsilon, j}$ satisfies $x_{\varepsilon, j, 1}=$ $\psi\left(x_{\varepsilon, j}^{\prime \prime}\right)-\gamma$, or $x_{\varepsilon, j} \in \partial B_{\delta^{\prime}}(\bar{x})$, or that there is a $j \in\left\{k_{1}+1, \ldots, k_{1}+k_{2}\right\}$, such that $x_{j} \in \partial D \cap \partial B_{\delta}(\bar{x})$, then $x_{\varepsilon, j, 1} \leq \bar{x}_{1}-\beta$ for some small $\beta>0$. So, by (3.39),

$$
K\left(x_{\varepsilon}\right) \leq A\left(k_{1}+k_{2} / 2\right) \varepsilon^{N-m+1} \bar{x}_{1}^{m-1}-c^{\prime} \varepsilon^{N-m+1}+\varepsilon^{N-m+1} O(\varepsilon)<K\left(\bar{x}_{\varepsilon}\right),
$$

where $c^{\prime}>0$ is a small constant. This is a contradiction.

Suppose that there are $i, j \in\left\{1, \ldots, k_{1}+k_{2}\right\}, i \neq j$, such that $e^{-\left|x_{i}-x_{j}\right| / \varepsilon}=$ $\varepsilon^{1-\tilde{\theta}}$. Then

$$
K\left(x_{\varepsilon}\right) \leq A\left(k_{1}+k_{2} / 2\right) \varepsilon^{N-m+1} \bar{x}_{1}^{m-1}-c_{0} \varepsilon^{N-m+2-\tilde{\theta}}+O\left(\varepsilon^{N-m+2}\right)<K\left(\bar{x}_{\varepsilon}\right) .
$$

This is also a contradiction.

So $x_{\varepsilon}$ is an interior point of $D_{\varepsilon}$. As a result,

$$
\begin{aligned}
D_{x_{i, h}} K\left(x_{\varepsilon}\right) & =0, i=1, \ldots, k_{1} ; h=1, \ldots, N-m+1 \\
D_{\tau_{j, l}} K\left(x_{\varepsilon}\right) & =0, j=k_{1}+1, \ldots, k_{1}+k_{2} ; l=1, \ldots, N-m,
\end{aligned}
$$

where

$$
\begin{aligned}
D_{x_{i, h}} K\left(x_{\varepsilon}\right) & =\frac{\partial J}{\partial x_{i, h}}+\left\langle\frac{\partial J}{\partial \omega}, \frac{\partial \omega}{\partial x_{i, h}}\right\rangle_{D, \varepsilon} \\
D_{\tau_{j, l}} K\left(x_{\varepsilon}\right) & =\frac{\partial J}{\partial \tau_{j, l}}+\left\langle\frac{\partial J}{\partial \omega}, \frac{\partial \omega}{\partial \tau_{j, l}}\right\rangle_{D, \varepsilon}
\end{aligned}
$$


On the other hand,

$$
\begin{aligned}
\left\langle\frac{\partial J}{\partial \omega}, \frac{\partial \omega}{\partial x_{i, h}}\right\rangle_{D, \varepsilon} & =\sum_{k=1}^{N-m+1} A_{i, k}\left\langle\frac{\partial P_{\varepsilon, \Omega} W_{\varepsilon, x_{i}}}{\partial x_{i, k}}, \frac{\partial \omega}{\partial x_{i, h}}\right\rangle_{D, \varepsilon} \\
& =-\sum_{k=1}^{N-m+1} A_{i, k}\left\langle\frac{\partial^{2} P_{\varepsilon, \Omega} W_{\varepsilon, x_{i}}}{\partial x_{i, k} \partial x_{i, h}}, \omega\right\rangle_{D, \varepsilon} \\
\left\langle\frac{\partial J}{\partial \omega}, \frac{\partial \omega}{\partial \tau_{j, l}}\right\rangle_{D, \varepsilon} & =\sum_{k=1}^{N-m} A_{j, k}\left\langle\frac{\partial P_{\varepsilon, \Omega} W_{\varepsilon, x_{j}}}{\partial \tau_{j, k}}, \frac{\partial \omega}{\partial \tau_{j, l}}\right\rangle_{D, \varepsilon} \\
& =-\sum_{k=1}^{N-m} A_{j, k}\left\langle\frac{\partial^{2} P_{\varepsilon, \Omega} W_{\varepsilon, x_{j}}}{\partial \tau_{j, k} \partial \tau_{j, l}}, \omega\right\rangle_{D, \varepsilon}
\end{aligned}
$$

Therefore,

$$
\begin{aligned}
\frac{\partial J}{\partial x_{i, h}} & =\sum_{k=1}^{N-m+1} A_{i, k}\left\langle\frac{\partial^{2} P_{\varepsilon, \Omega} W_{\varepsilon, x_{i}}}{\partial x_{i, k} \partial x_{i, h}}, \omega\right\rangle_{D, \varepsilon}, \\
\frac{\partial J}{\partial \tau_{j, l}} & =\sum_{k=1}^{N-m} A_{j, k}\left\langle\frac{\partial^{2} P_{\varepsilon, \Omega} W_{\varepsilon, x_{j}}}{\partial \tau_{j, k} \partial \tau_{j, l}}, \omega\right\rangle_{D, \varepsilon}
\end{aligned}
$$

which is exactly (3.2) and (3.3).

Using Lemma 3.1,

$$
\sum_{j=1}^{k_{1}+k_{2}} P_{\varepsilon, \Omega} W_{\varepsilon, x_{\varepsilon, j}}+\omega_{\varepsilon, x_{\varepsilon}}
$$

is a solution of (1.7). Since $f(y, t)=0$ if $t \leq 0$, we see that

$$
\sum_{j=1}^{k} P_{\varepsilon, \Omega} W_{\varepsilon, x_{\varepsilon, j}}+\omega_{\varepsilon, x_{\varepsilon}}
$$

is positive. But $\omega_{\varepsilon, x_{\varepsilon}} \in S_{\varepsilon}$. Thus $\left|\omega_{\varepsilon, x_{\varepsilon}}\right| \leq \frac{1}{2}$ in $\Omega \backslash B$, which gives

$$
f\left(y, \sum_{j=1}^{k_{1}+k_{2}} P_{\varepsilon, \Omega} W_{\varepsilon, x_{\varepsilon, j}}+\omega_{\varepsilon, x_{\varepsilon}}\right)=\left(\sum_{j=1}^{k_{1}+k_{2}} P_{\varepsilon, \Omega} W_{\varepsilon, x_{\varepsilon, j}}+\omega_{\varepsilon, x_{\varepsilon}}\right)_{+}^{p-1} .
$$

As a result,

$$
\sum_{j=1}^{k_{1}+k_{2}} P_{\varepsilon, \Omega} W_{\varepsilon, x_{\varepsilon, j}}+\omega_{\varepsilon, x_{\varepsilon}}
$$

is a solution of (1.1). 


\section{Appendix}

Lemma 4.1. Suppose $x_{i}, x_{j} \in D, x_{i} \neq x_{j}$ and $l, n=1, \ldots, N-m+1$, $x_{h}, x_{k} \in \partial D, x_{h} \neq x_{k}, r, \mu=1, \ldots, N-m$, we have

$$
\begin{aligned}
& \left\langle\frac{\partial P_{\varepsilon, \Omega} W_{\varepsilon, x_{j}}}{\partial x_{j, l}}, \frac{\partial P_{\varepsilon, \Omega} W_{\varepsilon, x_{j}}}{\partial x_{j, l}}\right\rangle_{D, \varepsilon}=C_{1} \varepsilon^{N-m-1}+O\left(\varepsilon^{N-m-1} e^{-\frac{c}{\varepsilon}}\right), \\
& \left\langle\frac{\partial P_{\varepsilon, \Omega} W_{\varepsilon, x_{j}}}{\partial x_{j, l}}, \frac{\partial P_{\varepsilon, \Omega} W_{\varepsilon, x_{i}}}{\partial x_{i, n}}\right\rangle_{D, \varepsilon}=O\left(\varepsilon^{N-m-1} e^{-\frac{\left|x_{i}-x_{j}\right|}{\varepsilon}}+\varepsilon^{N-m-1} e^{-\frac{c}{\varepsilon}}\right), \\
& \left\langle\frac{\partial P_{\varepsilon, \Omega} W_{\varepsilon, x_{j}}}{\partial x_{j, l}}, \frac{\partial P_{\varepsilon, \Omega} W_{\varepsilon, x_{j}}}{\partial x_{j, n}}\right\rangle_{D, \varepsilon}=O\left(\varepsilon^{N-m-1}\right), \quad l \neq n, \\
& \left\langle\frac{\partial P_{\varepsilon, \Omega} W_{\varepsilon, x_{h}}}{\partial \tau_{h, r}}, \frac{\partial P_{\varepsilon, \Omega} W_{\varepsilon, x_{h}}}{\partial \tau_{h, r}}\right\rangle_{D, \varepsilon}=C_{2} \varepsilon^{N-m-1}+O\left(\varepsilon^{N-m-1} e^{-\frac{c}{\varepsilon}}\right), \\
& \left\langle\frac{\partial P_{\varepsilon, \Omega} W_{\varepsilon, x_{h}}}{\partial \tau_{h, r}}, \frac{\partial P_{\varepsilon, \Omega} W_{\varepsilon, x_{k}}}{\partial \tau_{k, \mu}}\right\rangle_{D, \varepsilon}=O\left(\varepsilon^{N-m-1} e^{-\frac{\left|x_{h}-x_{k}\right|}{\varepsilon}}+\varepsilon^{N-m-1} e^{-\frac{c}{\varepsilon}}\right), \\
& \left\langle\frac{\partial P_{\varepsilon, \Omega} W_{\varepsilon, x_{h}}}{\partial \tau_{h, r}}, \frac{\partial P_{\varepsilon, \Omega} W_{\varepsilon, x_{h}}}{\partial \tau_{h, \mu}}\right\rangle_{D, \varepsilon}=O\left(\varepsilon^{N-m-1}\right), \quad r \neq \mu, \\
& \left\langle\frac{\partial P_{\varepsilon, \Omega} W_{\varepsilon, x_{i}}}{\partial x_{i, l}}, \frac{\partial P_{\varepsilon, \Omega} W_{\varepsilon, x_{h}}}{\partial \tau_{h, \mu}}\right\rangle_{D, \varepsilon}=O\left(\varepsilon^{N-m-1} e^{-\frac{\left|x_{i}-x_{h}\right|}{\varepsilon}}+\varepsilon^{N-m-1} e^{-\frac{c}{\varepsilon}}\right),
\end{aligned}
$$

where $C_{1}, C_{2}>0$ are constants.

Proof. The proof is similar to [?] and [6] and we omit it here.

\section{References}

[1] Ambrosetti, A., Malchiodi, A. and Ni, W.-M.: Singularly perturbed elliptic equations with symmetry: existence of solutions concentrating on spheres. I. Comm. Math. Phys. 235 (2003), 427-466.

[2] Ambrosetti, A., Malchiodi, A. And Ni, W. M.: Singularly perturbed elliptic equations with symmetry: existence of solutions concentrating on spheres. II. Indiana Univ. Math. J. 53 (2004), 297-329.

[3] Bartsch, T. And Peng, S.: Existence of solutions concentrating on higher dimensional subsets for singularly perturbed elliptic equations (I). To appear in Indiana Univ. Math. J.

[4] Bartsch, T. And Peng, S.: Existence of solutions concentrating on higher dimensional subsets for singularly perturbed elliptic equations (II). Preprint.

[5] Bates, P., Dancer, E. N. And Shi, J.: Multi-spike stationary solutions of the Cahn-Hilliard equation in higher-dimension and instability. Adv. Differential Equations 4 (1999), 1-69. 
[6] CaO, D. And KÜpper, T.: On the existence of multipeaked solutions to a semilinear Neumann problem Duke Math. J. 97 (1999), 261-300.

[7] Dancer, E. N.: Some singularly perturbed problems on annuli and a counterexample to a problem of Gidas, Ni and Nirenberg. Bull. London Math. Soc. 29 (1997), 322-326.

[8] Dancer, E. N. and Yan, S.: Multipeak solutions for a singularly perturbed Neumann Problem. Pacific J. Math. 189 (1999), 241-262.

[9] Dancer, E. N. And YAN, S.: Interior and boundary peak solutions for a mixed boundary value problem. Indiana Univ. Math. J. 48 (1999), 11771212 .

[10] Dancer, E. N. and Yan, S.: A new type of concentration solutions for a singularly perturbed elliptic problem. Trans. Amer. Math. Soc. 359 (2007), no. $4,1765-1790$.

[11] Del Pino, M. And Felmer, P.: Spike-layered solutions of singularly perturbed elliptic problems in a degenerate setting. Indiana Univ. Math. J. 48 (1999), 883-898.

[12] Gui, C. AND WeI, J.: Multiple interior peak solutions for some singularly perturbed Neumann problems. J. Differential Equations 158 (1999), 1-27.

[13] Gui, C. AND Wei, J.: On multiple mixed interior and boundary peak solutions for some singularly perturbed Neumann problems. Canad. J. Math. 52 (2000), 522-538.

[14] Gui, C., Wei, J. And Winter, M.: Multiple boundary peak solutions for some singularly perturbed Neumann problems. Ann. Inst. H. Poincaré Anal. Non Linéaire 17 (2000), 47-82.

[15] KWOnG, M. K.: Uniqueness of positive solutions of $\Delta u-u+u^{p}=0$ in $R^{n}$. Arch. Rational Mech. Anal. 105 (1989), 243-266.

[16] LI, Y.Y.: On a singularly perturbed equation with Neumann boundary condition. Comm. Partial Differential Equations 23 (1998), 487-545.

[17] Malchiodi, A.: Construction of multidimensional spike-layers. Discrete Contin. Dyn. Syst. 14 (2006), 187-202.

[18] Malchiodi, A. And Montenegro, M.: Boundary concentration phenomena for a singularly perturbed elliptic problem. Comm. Pure Appl. Math. 55 (2002), 1507-1568.

[19] Malchiodi, A. And Montenegro, M.: Multidimensional boundary layers for a singularly perturbed Neumann problem. Duke Math. J. 124 (2004), $105-143$.

[20] Malchiodi, A., Ni, W.-M. And Wei, J.: Multiple clustered layer solutions for semilinear Neumann problems on a ball. Ann. Inst. H. Poincaré Anal. Non Linéaire 22 (2005), 143-163.

[21] NI, W.-M.: Diffusion, cross-diffusion, and their spike-layer steady states. Notices Amer. Math. Soc. 45 (1998), 9-18. 
[22] Ni, W.-M. And Takagi, I.: On the shape of least-energy solutions to a semilinear Neumann problem. Comm. Pure Appl. Math. 44 (1991), 819851.

[23] Ni, W.-M. AND TAKAGI, I.: Locating the peaks of least-energy solutions to a semilinear Neumann problem. Duke Math. J. 70 (1993), 247-281.

[24] WEI, J.: On the boundary spike layer solutions to a singularly perturbed Neumann problem. J. Differential Equations 134 (1997), 104-133.

[25] YAN, S.: On the number of interior multipeak solutions for singularly perturbed Neumann problems. Topol. Methods Nonlinear Anal. 12 (1998), $61-78$.

Recibido: 29 de mayo de 2006

Gongbao Li

School of Mathematics and Statistics

Central China Normal University

Wuhan, 430079, P. R. China

ligb@mail.ccnu.edu.cn

Shuangjie Peng

School of Mathematics and Statistics

Central China Normal University

Wuhan, 430079, P. R. China

sjpeng@mail.ccnu.edu.cn

Shusen Yan

School of Mathematics, Statistics and Computer Science University of New England

Armidale, NSW 2351, Australia syan@turing. une.edu . au

The first author is partially supported by NSFC $(10571069,10631030)$. The second author is supported by the Alexander von Humboldt Foundation in Germany, the Key Project of Chinese Ministry of Education (107081) and NCET-07-0350. 